\title{
Signal Transduction in Light-Oxygen-Voltage Receptors Lacking the Active-Site Glutamine
}

Julia Dietler

University of Bayreuth https://orcid.org/0000-0002-0418-0796

Renate Gelfert

University of Bayreuth

Jennifer Kaiser

University of Bayreuth

Veniamin Borin

Hebrew University Jerusalem https://orcid.org/0000-0001-7832-1443

Christian Renzl

Universität Bonn

Sebastian PilsI

University of Bonn

Américo Tavares Ranzani

University of Bayreuth

Andrés García de Fuentes

University of Bayreuth

Tobias Gleichmann

Humboldt University Berlin

Ralph Diensthuber

Humboldt University Berlin

Michael Weyand

University of Bayreuth

Günter Mayer

University of Bonn https://orcid.org/0000-0003-3010-4049

Igor Schapiro

Hebrew University Jerusalem https://orcid.org/0000-0001-8536-6869

Andreas Möglich ( $\nabla$ andreas.moeglich@uni-bayreuth.de)

University of Bayreuth https://orcid.org/0000-0002-7382-2772

Article 
Keywords: glutamine, hydrogen bond, light-oxygen-voltage, optogenetics, photoreception, signal transduction

Posted Date: October 26th, 2021

DOI: https://doi.org/10.21203/rs.3.rs-956213/v1

License: (c) (1) This work is licensed under a Creative Commons Attribution 4.0 International License. Read Full License

Version of Record: A version of this preprint was published at Nature Communications on May 12th, 2022. See the published version at https://doi.org/10.1038/s41467-022-30252-4. 


\section{Signal Transduction in Light-Oxygen-Voltage Receptors Lacking the Active-Site Glutamine}

Julia Dietler ${ }^{1, \#}$, Renate Gelfert ${ }^{1, \#}$, Jennifer Kaiser ${ }^{1, \#}$, Veniamin Borin ${ }^{2}$, Christian Renzl $^{3}$, Sebastian Pils| ${ }^{3}$, Américo Tavares Ranzani ${ }^{1}$, Andrés García de Fuentes ${ }^{1}$, Tobias Gleichmann ${ }^{4}$, Ralph P. Diensthuber ${ }^{4}$, Michael Weyand ${ }^{1}$, Günter Mayer ${ }^{3,5}$, Igor Schapiro ${ }^{2}$, and Andreas Möglich ${ }^{1,4,6,7,+, *}$

${ }^{1}$ Department of Biochemistry, University of Bayreuth, 95447 Bayreuth, Germany

${ }^{2}$ Institute of Chemistry, The Hebrew University of Jerusalem, Jerusalem, Israel

${ }^{3}$ Life and Medical Sciences (LIMES), University of Bonn,53121 Bonn, Germany

${ }^{4}$ Biophysical Chemistry, Humboldt-University Berlin, 10115 Berlin, Germany

${ }^{5}$ Center of Aptamer Research \& Development, University of Bonn, 53121 Bonn, Germany

${ }^{6}$ Bayreuth Center for Biochemistry \& Molecular Biology, Universität Bayreuth, 95447 Bayreuth, Germany

${ }^{7}$ North-Bavarian NMR Center, Universität Bayreuth, 95447 Bayreuth, Germany

\# These authors contributed equally.

† ORCID identifiers: J.D. 0000-0002-0418-0796; R.G. 0000-0002-2677-5774; J.K. 0000-0003-3029-3505; V.B. 0000-0001-7832-1443; C.R. 0000-0003-2296-1825; S.P. 0000-0003-1063-1407; A.T.R. 0000-0002-6203-9467; A.G.F. 0000-0002-0072-4725; R.P.D. 0000-0002-4864-7001; M.W. 0000-0002-7499-1324; G.M. 0000-00033010-4049; I.S. 0000-0001-8536-6869; A.M. 0000-0002-7382-2772

* To whom correspondence should be addressed. Tel: +49-921-55-7835; Email: andreas.moeglich@uni-bayreuth.de

\section{Abstract}

In nature as in biotechnology, light-oxygen-voltage (LOV) photoreceptors perceive blue light to elicit spatiotemporally defined cellular responses. Photon absorption drives thioadduct formation between a conserved cysteine and the flavin chromophore. An equally conserved, proximal glutamine processes the resultant flavin protonation into downstream hydrogen-bond rearrangements. Here, we report that this glutamine, long deemed essential, is generally dispensable. In its absence, several LOV receptors invariably retained productive, if often attenuated, signaling responses. Structures of a LOV paradigm at around 1 Å resolution revealed highly similar light-induced conformational changes, irrespective of whether the glutamine is present. Naturally occurring, glutamine-deficient LOV receptors likely serve as bona fide photoreceptors, as we showcase for a diguanylate cyclase. We propose that without the glutamine, water molecules transiently approach the chromophore and thus propagate flavin protonation downstream. Signaling without glutamine appears intrinsic to LOV receptors, which pertains to biotechnological applications and suggests evolutionary descendance from redox-active flavoproteins. 


\section{Keywords}

glutamine; hydrogen bond; light-oxygen-voltage; optogenetics; photoreception; signal transduction

\section{Introduction}

Light-oxygen-voltage (LOV) proteins form a sensory photoreceptor class that elicit a wide palette of physiological responses to blue light across archaea, bacteria, protists, fungi, and plants ${ }^{1-3}$. Complementing their eminent role in nature, LOV receptors also serve as genetically encoded actuators in optogenetics ${ }^{4}$ for the spatiotemporally precise control by light of cellular state and processes ${ }^{5}$. At the heart of these responses lies the flavin-binding LOV photosensor module which belongs to the Per-ARNT-Sim superfamily ${ }^{6}$ and comprises several $\alpha$ helices (denoted $\mathrm{C} \alpha, \mathrm{D} \alpha, \mathrm{E} \alpha$, and $\mathrm{F} \alpha$ ) arranged around a five-stranded antiparallel $\beta$ sheet (strands $A \beta, B \beta, G \beta, H \beta$, and $I \beta)^{7,8}$ (Suppl. Fig. S1). Light absorption by the flavin triggers a well-studied photocycle ${ }^{2,9-}$ ${ }^{11}$, as part of which an initial electronically excited singlet state $\left(S_{1}\right)$ decays within nanoseconds to a triplet state $\left(T_{1}\right)$ (Fig. 1a). Likely via radical-pair mechanism ${ }^{12}, T_{1}$ reacts within microseconds to the signaling state, characterized by a covalent thioadduct between a highly conserved cysteine residue in the LOV photosensor and the $\mathrm{C} 4 \mathrm{a}$ atom of the flavin isoalloxazine ring system. Once illumination ceases, the signaling state passively reverts to the resting state in the base-catalyzed dark-recovery reaction ${ }^{13}$. Thioadduct formation entails a hybridization change of the flavin C4a atom from $s p^{2}$ to $s p^{3}$ and concomitant protonation of the adjacent N5 atom. The resultant conversion of the N5 position from a hydrogen bond acceptor to a donor serves as the principal trigger ${ }^{14}$ for a raft of conformational and dynamic transitions, that depending upon LOV receptor, culminate in order-disorder transitions ${ }^{15}$, oligomerization ${ }^{16}$, or other quaternary structural changes ${ }^{17}$. A strictly conserved glutamine residue in strand $I \beta$ is situated immediately adjacent to the flavin and has been identified as instrumental in reading out the flavin N5 position and eliciting the downstream transitions. Supported by spectroscopy, structural and functional data, chemical reasoning, and molecular simulations ${ }^{8,18-23}$, the glutamine is widely held to rotate its amide sidechain to accommodate $\mathrm{N} 5$ protonation in the signaling state. As a corollary, additional hydrogen-bond rearrangements permeate the LOV photosensor and propagate towards the $\beta$-sheet scaffold. As recently proposed ${ }^{24}$, glutamine reorientation and signal propagation may be aided by transient rearrangements of two conserved asparagine residues that coordinate the pteridin portion of the flavin. 


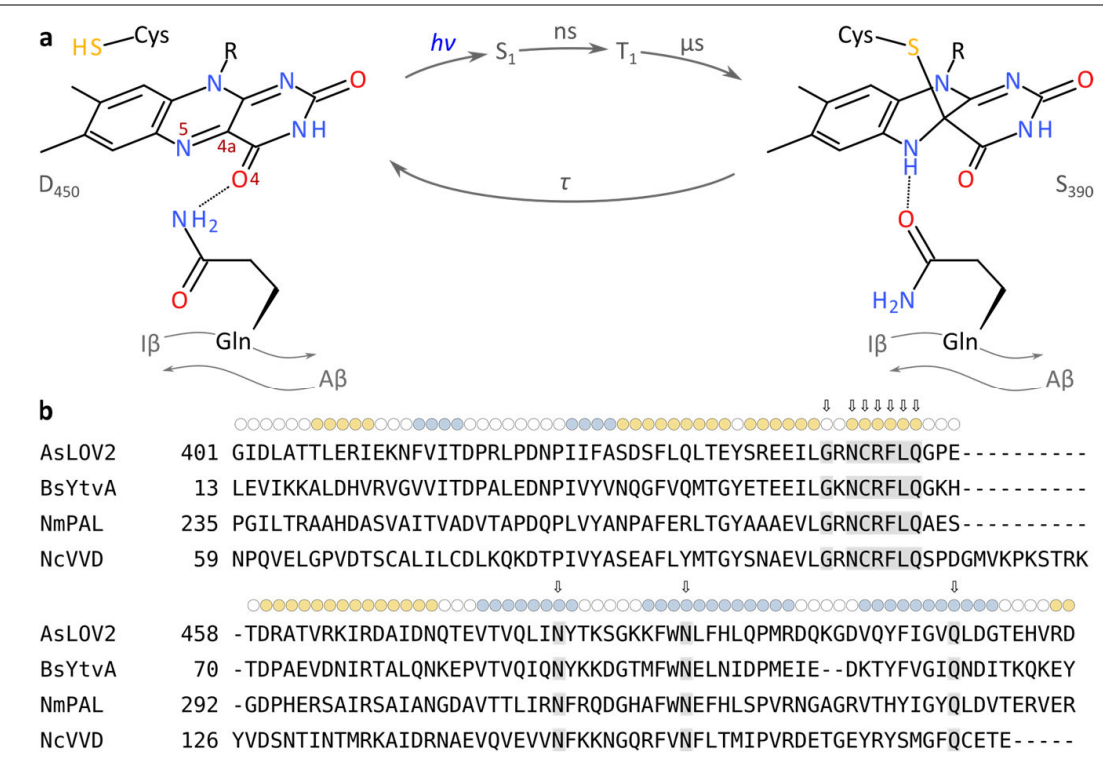

Fig. 1 - a, Photocycle of light-oxygen-voltage (LOV) receptors. Absorption of blue light by the darkadapted state $\left(D_{450}\right)$ prompts the LOV receptor to traverse short-lived excited singlet $\left(S_{1}\right)$ and triplet $\left(T_{1}\right)$ states before assuming the light-adapted state $\left(\mathrm{S}_{390}\right)$ which is characterized by a thioadduct between the flavin atom $\mathrm{C} 4 \mathrm{a}$ and the sidechain of a conserved cysteine. Adduct formation goes along with protonation of the N5 atom which entails changes in hydrogen bonding within the LOV receptor, particularly of a conserved glutamine residue situated in strand $I \beta$ of an antiparallel $\beta$ pleated sheet. The light-adapted state passively decays to the dark-adapted state over a matter of seconds to hours, depending on the flavin surroundings. $\mathbf{b}$, Multiple sequence alignment of $A$. sativa phototropin 1 LOV2 (AsLOV2) ${ }^{15}$, B. subtilis YtvA LOV (BsYtvA) ${ }^{25}$, N. multipartita PAL LOV (NmPAL) ${ }^{26}$, and N. crassa Vivid LOV (NcVVD) ${ }^{27}$. The secondary structure, as observed in AsLOV2 ${ }^{28}$, is indicated on top, with $\alpha$ helices in tan and $\beta$ strands in blue. Residues conserved across LOV receptors ${ }^{29}$ are highlighted by arrows and grey shading.

Notwithstanding the strict conservation of the glutamine residue and its established role in LOV receptors, recent reports indicate that at least in certain proteins, productive signaling responses to blue light may gen-bonding changes as a means of signal transduction ${ }^{30,33}$. By contrast, reports on other LOV receptors considered the glutamine essential for eliciting blue-light responses ${ }^{21,34}$. To rationalize these conflicting findings and to provide further insight into signal transduction, we systematically investigated the role of the conserved glutamine in several model LOV receptors (Fig. 1b and Suppl. Fig. S1). Unexpectedly, the glutamine residue is not essential in LOV signaling as productive blue-light responses were generally maintained even in its absence. Almost all other amino acids could functionally substitute for the conserved glutamine, with notable exceptions. High-resolution crystal structures of the paradigm Avena sativa phototropin 1 LOV2 (AsLOV2) domain revealed that after glutamine substitution by leucine, closely similar structural changes are evoked by light as in the wild type. Based on structural data, chemical reasoning, and molecular simulations, we propose that in the absence of the glutamine, water molecules relay hydrogen-bonding signals from the flavin N5 position to the LOV $\beta$ sheet. The ability to transduce light signals without the glutamine appears to 
be an inherent, general trait of LOV receptors and may reflect their evolutionary origin. This notion finds support in the existence in nature of numerous LOV receptors that lack the conserved glutamine and presumably serve as blue-light receptors, as we confirm for a glutamine-deficient, proteobacterial LOV-diguanylate cyclase.

\section{Results}

\section{Signal transduction in LOV receptors lacking the active-site glutamine.}

To evaluate if and how LOV photosensors can transduce light signals to associated effector units in the absence of the conserved glutamine, we initially resorted to the histidine kinase YF1, as it allows the efficient assessment of signaling responses ${ }^{35-37}$. Together with the response regulator BjFixJ, the engineered LOV receptor YF1 forms a light-sensitive two-component system (TCS) (Fig. 2a). E. coli cultures harboring the pDusk-DsRed plasmid ${ }^{35}$, which encodes the YF1/BjFixJ TCS, exhibited strong expression of the red-fluorescent reporter DsRed as YF1 acts as a net kinase in darkness ${ }^{37}$. Blue light converts YF1 to a net phosphatase, and accordingly the DsRed fluorescence decreased by around 12-fold (Fig. 2b).
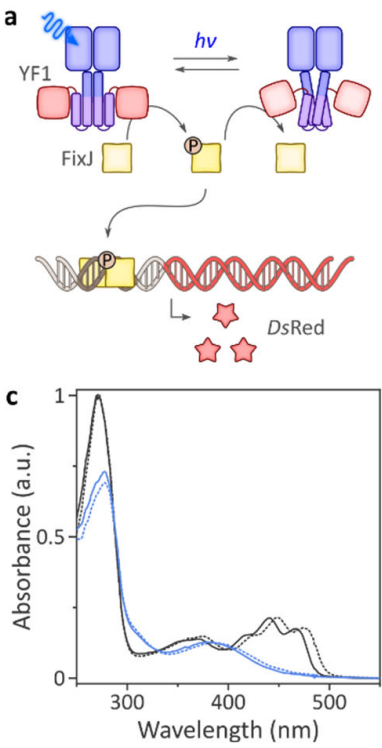

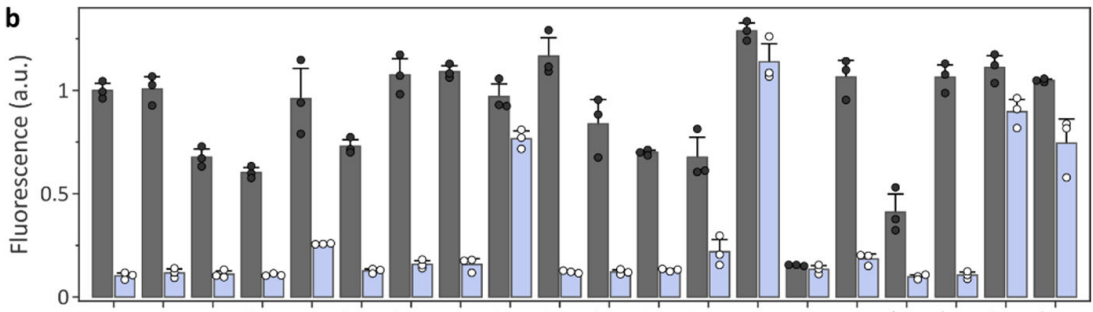

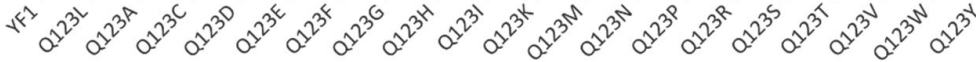
d
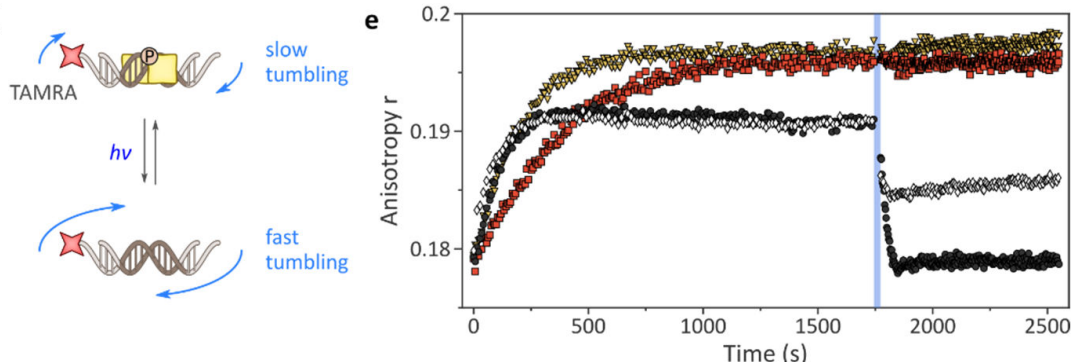

Fig. 2 - Activity and light response of YF1 variants. a, The net kinase activity of the variants was assessed in the pDusk-DsRed setup ${ }^{35}$, where alongside the response regulator BjFixJ, YF1 drives the expression of the red-fluorescent reporter DsRed in blue-light-repressed manner. $\mathbf{b}$, Normalized DsRed fluorescence of $E$. coli cultures harboring pDusk plasmids encoding different YF1 variants. Cells were cultivated in darkness (black dots and grey bars) or under constant blue light (white dots and blue bars). Data represent mean \pm s.d. of three biologically independent replicates. c, Absorbance spectra of YF1 Q123L (solid lines) in its dark-adapted (black) and light-adapted states (blue), compared to the corresponding spectra of YF1 (dotted lines). $\mathbf{d}$, Schematic of the coupled fluorescence anisotropy assay to probe YF1 activity. Once phosphorylated in light-dependent manner (see panel a), BjFixJ homodimerizes and binds to its cognate DNA operator sequence. Said operator is embedded in a TAMRA-labelled double-stranded DNA molecule, and BjFixJ binding can be detected as an increase in fluorescence anisotropy due to decelerated rotational tumbling. e, YF1 (black dots), YF1 Q123L (white diamonds), YF1 
Q123H (yellow triangles), or YF1 Q123P (red squares) were incubated in darkness together with BjFixJ and the TAMRA-labelled DNA. At time zero, the reaction was initiated by ATP addition and fluorescence anisotropy was recorded for $30 \mathrm{~min}$. Samples were then illuminated for $30 \mathrm{~s}$ with blue light (blue bar), and the measurement continued. All experiments were repeated at least twice with similar results.

To probe the role of the active-site glutamine (position Q123) in signal transduction, we substituted this residue for all 19 other canonical amino acids. Strikingly, most of the resultant glutamine-deficient YF1 variants prompted a blue-light-induced reduction of reporter gene fluorescence, similar to the original YF1 and almost regardless of which residue replaced the glutamine. These data clearly indicate that at least in the pDusk setup, the majority of residue substitutions, including alanine, cysteine, glutamic acid and leucine, leave light-dependent signal transduction largely unimpaired. Merely, the substitution by proline and the bulky aromatic amino acids His, Trp, and Tyr abolished responsiveness and resulted in high reporter expression independently of light. Similarly, the Q123R variant did not react to light but exhibited constitutively low reporter fluorescence.

To glean additional insight, we expressed and purified the variants Q123H, Q123L, Q123P, and Q123R alongside YF1. Absorbance spectroscopy revealed flavin incorporation, as indicated by a three-pronged peak around $450 \mathrm{~nm}$, for all variants but Q123R which failed to incorporate the chromophore and was prone to aggregation (Fig. 2c and Suppl. Fig. S2). As indicated by circular dichroism (CD) spectroscopy, the variants Q123H, Q123L, and Q123P were folded and adopted secondary and by inference, tertiary structure similar to YF1 (Suppl. Fig. S2). Upon blue-light exposure, YF1 and its variants Q123L and Q123P underwent the canonical LOV photocycle and adopted the thioadduct state with a characteristic absorption maximum near $390 \mathrm{~nm}$ (Fig. 2c and Suppl. Fig. S2). By contrast, the Q123H variant failed to form the adduct state despite incorporating the flavin cofactor, in line with earlier reports on AsLOV2 ${ }^{38}$. Only at high blue-light doses, the flavin absorption band slightly decreased in intensity but no band at $390 \mathrm{~nm}$ was formed. As reported earlier 20,21,38, replacement of the glutamine residue incurred a hypsochromic shift by around $8 \mathrm{~nm}$ of the flavin absorbance peak in both the dark-adapted and light-adapted states. This spectral shift can tentatively be attributed to the loss of hydrogen bonding to the flavin $\mathrm{O} 4$ atom (see Fig. 1a) and is reminiscent of a bathochromic shift of similar magnitude during the photocycle of the so-called 'sensors of blue light using flavin adenine dinucleotide' (BLUF) ${ }^{39,40}$. Taken together, the absorbance data account for the absent light responses in the pDusk context (see Fig. 2b) of the Q123H (no photocycle) and Q123R variants (no chromophore).

We next recorded the dark recovery after blue-light exposure and found the return to the darkadapted state 10-fold decelerated in Q123L relative to YF1 (Suppl. Fig. S2). The Q123P variant exhibited even slower kinetics that were not completed even after several days. Given that the Q123L variant principally retained the capability of transducing signals (see Fig. 2 b), we reasoned that modification of the active-glu- 
tamine provides an additional, little tapped means of altering recovery kinetics ${ }^{41}$ and thus modulating photosensitivity at photostationary state ${ }^{42}$. To explore this effect, we assessed the response of YF1 Q123L to pulsatile blue-light illumination ${ }^{43}$ in the pDawn system that derives from pDusk but exhibits inverted response to blue light ${ }^{35}$. The Q123L variant was toggled by much lower light doses than YF1, fully consistent with its retarded dark recovery (Suppl. Fig. S3). Compared to the V28I substitution, which also decelerates dark recovery by around 10 -fold ${ }^{41,43,44}$, the Q123L exchange was somewhat less sensitive to blue light. Combining the substitutions V28I and Q123L did not provide a further gain but slightly reduced the effective light sensitivity.

As the pDusk and pDawn systems only indirectly report on the molecular activity of the receptors, we probed the catalytic activity and response to light of purified YF1 and its variants in a coupled fluorescence anisotropy assay (Fig. 2d). In darkness and in the presence of ATP, YF1 phosphorylates its cognate response regulator BjFixJ, thus prompting its homodimerization and binding of the FixK2 DNA operator sequence ${ }^{14,45}$. Phosphorylation-induced binding of BjFixJ to a short, double-stranded DNA molecule slows its rotational diffusion and causes an increase in fluorescence anisotropy of a $5^{\prime}$-attached tetramethylrhodamine (TAMRA) moiety. As noted above, blue light converts YF1 into a net phosphatase, thus promoting BjFixJ dephosphorylation, DNA dissociation and a decrease of fluorescence anisotropy. Upon ATP addition, the dark-adapted YF1 and the Q123H, Q213L, and Q123P variants all exhibited increasing fluorescence anisotropy, albeit with somewhat differing kinetics and amplitude. Whereas Q123L showed similar response as YF1, the Q123H and Q123P variants reached higher anisotropy values which likely reflects a higher degree of $B j F i x J$ phosphorylation than the roughly $50 \%$ achieved for YF1 ${ }^{37}$. The intrinsic equilibrium between the elementary histidine kinase and phosphatase activities of the TCS thus appears tilted towards the kinase state for Q123H and Q123P compared to YF1 and Q123L ${ }^{46,47}$. Upon blue-light application, the Q123L variant responded with a rapid fluorescence anisotropy decay of around half the amplitude seen for YF1, indicating that light signals are transduced by this variant but less efficiently so (Fig. 2e). Consistent with the pDusk reporter assay (see Fig. 2b), neither the Q123H nor the Q123P variant showed any response in their catalytic activities to blue light. In case of $\mathrm{Q} 123 \mathrm{H}$, these observations are readily explained by its inability to undergo light-induced adduct formation and flavin N5 protonation. By contrast, the absorbance measurements unequivocally showed that Q123P can progress through the canonical LOV photocycle (see Suppl. Fig. S2). As the LOV photochemistry hence remains intact, signal transduction in the Q123P variant must be interrupted further downstream.

\section{LOV signal transduction can generally occur in the absence of the active-site glutamine.}

We next addressed whether the unexpected ability to transduce light signals without the conserved glutamine residue is specific for YF1 or more widely shared across LOV receptors. To this end, we examined lightdependent signaling responses in Nakamurella multipartita PAL ${ }^{26}$, as a naturally occurring LOV receptor, and the $A$. sativa phototropin 1 LOV2 domain, as the arguably best-studied and optogenetically most widely used 
LOV module 5,15,28,48. Notably, NmPAL differs from YF1 by an unusual C-terminal arrangement of its LOV photosensor and binds a small RNA aptamer sequence-specifically and in light-activated manner ${ }^{26}$. By embedding this aptamer directly upstream of the Shine-Dalgarno sequence in an mRNA encoding the fluorescent DsRed protein, NmPAL activity and response to light can be assessed in a bacterial reporter assay (Fig. 3a). In its dark-adapted state, wild-type NmPAL has little affinity for the aptamer, and DsRed is readily expressed. Light-induced binding by NmPAL interferes with expression, presumably at the translational level, and reporter fluorescence is diminished by 10-fold (Fig. 3b). Using this assay, we tested the effect of replacing the active-site glutamine (residue Q347 in NmPAL) by histidine, leucine, or proline. Consistent with the findings for YF1, the resultant Q347H and Q347P variants no longer exhibited light-induced changes in reporter fluorescence. As in the YF1 case, the proline variant had constitutive activity similar to the dark-adapted parental wild-type NmPAL. Conversely, for Q347H we observed constitutively low fluorescence values, indicative of RNA binding and thus corresponding to light-adapted wild-type NmPAL. This contrasts with YF1 where the corresponding histidine variant functionally corresponded to the dark-adapted state of the parental receptor. The Q347L variant exhibited a light-induced decrease of DsRed fluorescence by around 17-fold, thus even surpassing the value for wild-type NmPAL. Taken together, the results from the NmPAL reporter assay are broadly consistent with the findings for YF1 in that the leucine substitution supported light responses to significant extent whereas the histidine and proline substitutions incurred a loss of light-dependent signal transduction.

a
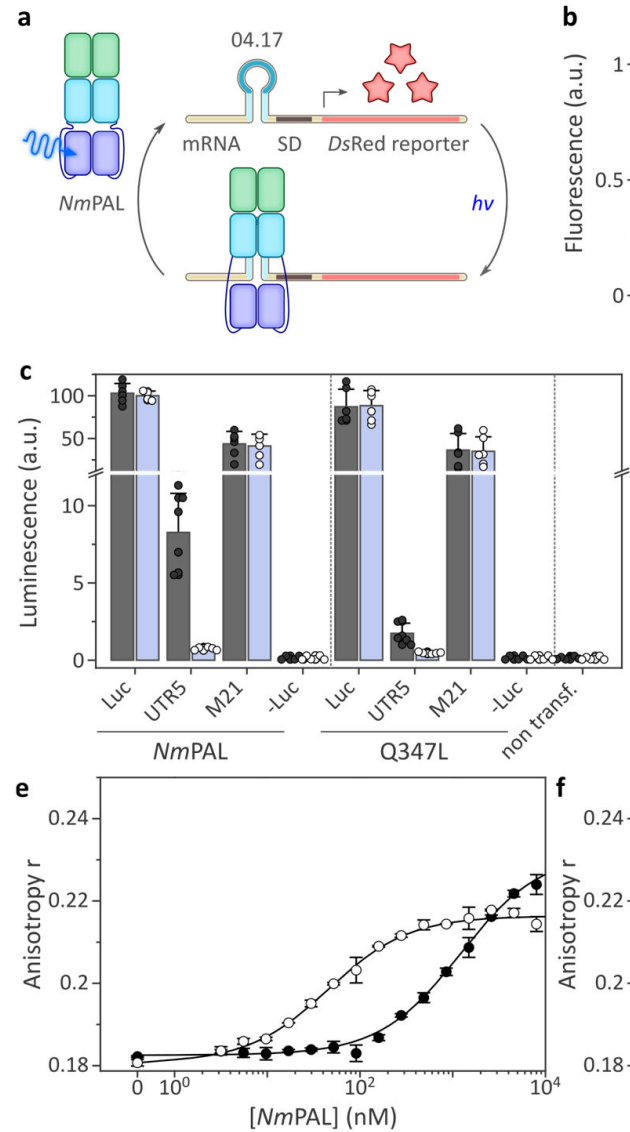

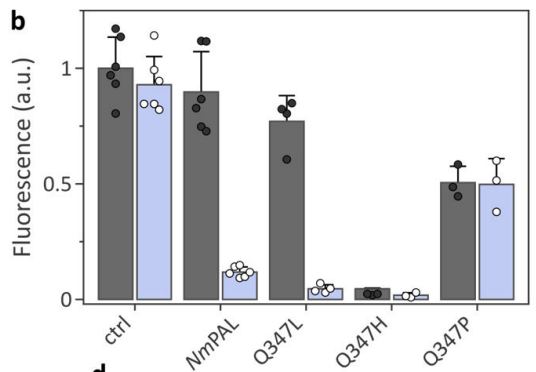
d

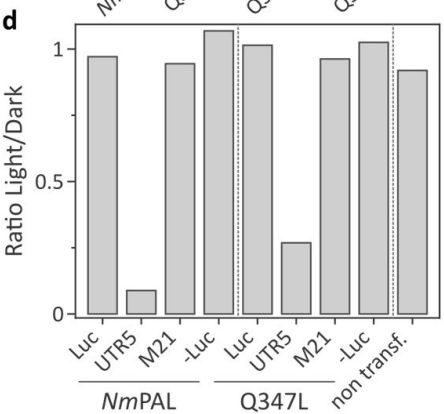

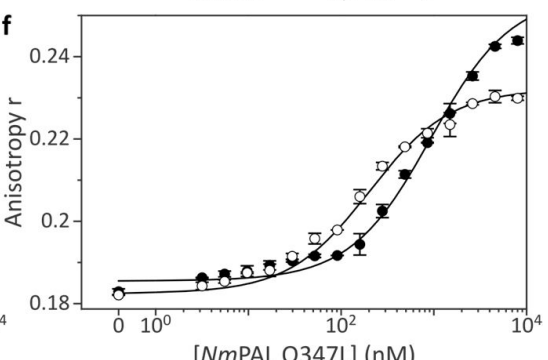


Fig. 3 - Activity and light response of NmPAL variants. a, By embedding a specific aptamer (denoted 04.17) near the Shine-Dalgarno sequence (SD) of an mRNA encoding DsRed, the expression of the fluorescent reporter can be modulated with $\mathrm{NmPAL}$ as a function of blue light ${ }^{26}$. In darkness, NmPAL shows little affinity for the aptamer, and expression ensues. Under blue light, NmPAL binds and thus

214

215

216

217

218

219

220

221

222

223

224 attenuates expression. b, E. coli cultures harboring different NmPAL variants and the reporter system depicted in panel a were cultivated in darkness (black dots and grey bars) or under blue light (white dots and blue bars). Normalized DsRed fluorescence represents mean \pm s.d. of at least three biologically independent samples. c, NmPAL variants were expressed in HeLa cells to translationally repress expression of a luciferase reporter, conceptually similar to the setup shown in panel a but using the 53.19 aptamer $^{26}$. Bars represent mean \pm s.d. of luminescence acquired for six biologically independent samples incubated in darkness (black dots and grey bars) or under blue light (white dots and blue bars). UTR5 refers to the intact reporter system giving rise to NmPAL-mediated light responses ${ }^{26}$; in M21, NmPAL binding is disrupted by a mutation in the target aptamer, and light responsiveness is abolished. As positive and negative controls, luciferase was constitutively expressed (Luc) or left out altogether (Luc). $\mathbf{d}$, Ratio of the luminescence values obtained under light and dark conditions. $\mathbf{e}$, The interaction of wild-type NmPAL with the TAMRA-labeled 04.17 aptamer was assessed in its dark-adapted (black dots) and light-adapted states (white dots) by fluorescence anisotropy ${ }^{26}$. The line represents a fit to a single-site binding isotherm. $f$, As in panel e but for NmPAL Q347L. Experiments in panels e and f were repeated twice with similar results.

We next tested whether the ability of NmPAL Q347L to transduce light signals extends to applications in eukaryotic cells. To this end, we harnessed an approach based on the translational repression of a luciferase reporter in HeLa cells ${ }^{26}$ (Fig. 3c). Under blue light, wild-type NmPAL can bind to an aptamer sequence embedded in the $5^{\prime}$-untranslated region of an mRNA and thereby represses luciferase expression by 10 -fold relative to darkness (Fig. 3d). Upon introduction of the Q347L substitution into NmPAL, blue-light-induced downregulation of reporter expression was maintained, albeit at reduced, 4-fold efficiency.

To investigate photochemistry and RNA binding in detail, we expressed and purified NmPAL wild-type and Q347L. In line with the reporter assays (see Fig. 3b-d), the Q347L variant retained flavin chromophore binding and underwent canonical LOV photochemistry upon blue-light exposure (Suppl. Fig. S4). As in YF1, replacement of the glutamine entailed a hypsochromic shift of the flavin absorption. Recovery kinetics after blue-light illumination were however only slowed down by 1.2-fold in the Q347L variant, rather than the 10fold slowdown in YF1. Far-UV CD spectroscopy showed that NmPAL and its Q347L variant adopt closely similar secondary structure (Suppl. Fig. S4c). We next assessed the binding of NmPAL wild-type and Q347L to a TAMRA-labelled RNA aptamer by fluorescence anisotropy ${ }^{26}$ (Fig. 3e, f). Wild-type NmPAL bound the RNA with an affinity of $(45.4 \pm 5.4) \mathrm{nM}$ in its light-adapted state but showed much reduced interaction in darkness [(1200 \pm 93$) \mathrm{nM}]$. Under the same conditions, NmPAL Q347L interacted with the aptamer somewhat less strongly under blue light [(202.5 \pm 8.5$) \mathrm{nM}$ ] but exhibited more pronounced residual binding in darkness with an affinity of around $(930 \pm 70) \mathrm{nM}$. Thus, light-dependent signal transduction is principally retained in NmPAL Q347L but is impaired compared to the wild-type receptor, similar to the observations made for YF1. 
We next turned to the LOV2 domain from A. sativa phototropin 1 (AsLOV2) as a widely studied para-

digm ${ }^{15,28,38,49,50}$ that underpins manifold applications in optogenetics 5,51,52. Whereas AsLOV2 wild-type, Q513H, and Q513L could all be produced with good yield and purity, the Q513P variant suffered from poor expression and severe aggregation, thus precluding its further analysis. The Q513H and Q513L variants incorporated flavin cofactors and exhibited a hypsochromically shifted absorbance spectrum compared to wild-type AsLOV2 (Suppl. Fig. S5), as seen for YF1 and NmPAL. Under blue light, the Q513L variant populated the thioadduct state which recovered to the resting state in darkness with kinetics around 22 -fold slower than those of the wild-type domain (Suppl. Fig. S5). By contrast, the Q513H variant failed to undergo the canonical LOV photochemistry, consistent with the YF1 and NMPAL scenarios. The dark-adapted wild-type, Q513H, and Q513L proteins showed closely similar far-UV CD spectra, characterized by two minima of the molar ellipticity per residue, $[\theta]_{\mathrm{MRW}}$, at around $208 \mathrm{~nm}$ and $220 \mathrm{~nm}$, and consistent with the mixed $\alpha \beta$ fold of AsLOV2 ${ }^{28}$ (Fig. 4 and Suppl. Fig. S5). Exposure to blue light diminished the amplitude of the minima by around 30-35\% for both AsLOV2 wild-type and Q513L, reflecting the unfolding of the N-terminal A' $\alpha$ and the C-terminal Ja helices ${ }^{50}$. However, given the relatively fast recovery of AsLOV2 wild-type (see Suppl. Fig. S5), significant return to the dark-adapted state is expected during the spectral scan (taking around $1 \mathrm{~min}$ ). We hence monitored the $\alpha$-helical CD signal at $(208 \pm 5) \mathrm{nm}$ immediately after withdrawal of blue light (Fig. 4b, d). The kinetic measurements revealed that the initial amplitude of the light-induced CD change in AsLOV2 Q513L was only half that in the wild-type protein. For both variants, the CD spectra fully recovered to their original states (Fig. 4) with kinetics matching those of the photochemical recovery probed by absorbance measurements (see above and Suppl. Fig. S5). In agreement with our findings, an earlier study reported lightinduced CD changes for the Q513L variant but at much reduced amplitude compared to wild-type AsLOV2 ${ }^{21}$. Taken together, our CD measurements suggest that glutamine replacement by leucine (but not by histidine) qualitatively, if not quantitatively, preserves light-induced signaling responses, fully consistent with the results on the other LOV receptors. 

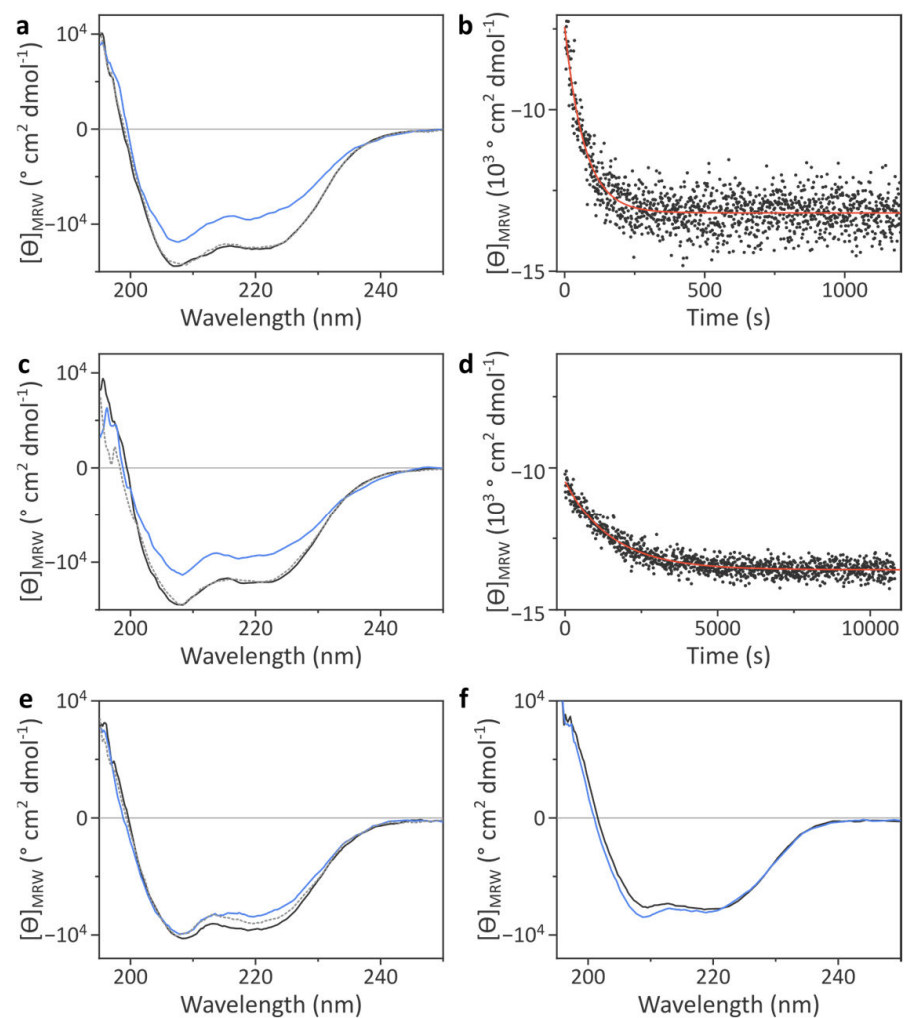

Fig. 4 - Light response of AsLOV2 variants. a, Far-UV circular dichroism (CD) spectra of AsLOV2 in its dark-adapted (black) and light-adapted states (blue), and after dark recovery (grey dotted). b, Recovery reaction of AsLOV2 following blue-light exposure, as monitored by the CD signal at $(220 \pm 5) \mathrm{nm}$. Data were fitted to a single-exponential decay (red line), yielding a recovery rate constant $k_{-1}$ of (1.43 $\pm 0.05) \times 10^{-2} \mathrm{~s}^{-1}$. c, As panel a but for AsLOV2 Q513L. d, As panel b but for AsLOV2 Q513L, with $k_{-1}$ amounting to $(6.61 \pm 0.15) \times 10^{-4} \mathrm{~s}^{-1}$. e, As panel a but for AsLOV2 C450A:Q513D. f, As panel a but for AsLOV2 C450A:Q513D $\Delta \mathrm{A}^{\prime} \alpha \Delta \mathrm{J} \alpha$. Experiments were repeated at least twice with similar results.

A previous investigation showed that LOV receptors can trigger productive signaling responses even when devoid of their active-site cysteine ${ }^{14}$. Blue light then promotes photoreduction of the flavin chromophore from its oxidized quinone form to the partially reduced neutral semiquinone (NSQ), which shares with the thioadduct state a protonated $\mathrm{N} 5$ atom and is thus capable of intact signal transduction ${ }^{14}$. We consequently wondered whether these aspects also hold true for LOV receptors that lack both the conserved cysteine and glutamine residues. As cysteine-deficient LOV receptors can efficiently sensitize molecular oxygen ${ }^{53}$, pertinent experiments may be complicated by reactive oxygen species (ROS) which potentially disrupt or obscure genuine signaling responses to blue light. We therefore opted to assess the effect of combined cysteine and glutamine removal by CD spectroscopy in the isolated AsLOV2 module, as a comparatively welldefined and tractable experimental setup. Replacement of the active-site cysteine (residue 450) in wild-type AsLOV2 by alanine abolished canonical photochemistry but the NSQ yield was poor, even at prolonged illumination and in the presence of the reductant TCEP (Suppl. Fig. S5). Nor did additional introduction of the Q513L exchange significantly enhance NSQ formation. We thus capitalized on the recent finding that replacement of the active-site glutamine by aspartate in an Arabidopsis thaliana phototropin LOV domain greatly 
promoted photoreduction to the NSQ ${ }^{54}$. Given that the corresponding Q123D substitution in YF1 retained signaling capability (see Fig. 2b), we generated AsLOV2 Q513D and the doubly substituted C450A:Q513D variant. Absorbance spectroscopy revealed that the Q513D variant underwent the canonical LOV photochemistry and formed the thioadduct state (Suppl. Fig. S5). CD spectroscopy showed a light-induced $25 \%$ loss of $[\theta]_{M R W}$, indicating that the Q513D variant can indeed transduce blue-light signals (Suppl. Fig. S5). In case of AsLOV2 C450A:Q513D, blue light drove rapid conversion to the NSQ state even without addition of reductants, as determined by absorbance spectroscopy (Suppl. Fig. S5). Analysis by CD spectroscopy identified an around $10 \%$ loss in $\alpha$-helical content upon blue-light exposure (Fig. 4e). Notably, the underlying conformational change was reversible, and upon slow reoxidation of the NSQ to the quinone state, the CD signal recovered over time. To ascertain that the change in helical content truly involves the $A^{\prime} \alpha$ and Ja helices as in wild-type AsLOV2, we generated the AsLOV2 C450A:Q513D $\Delta \mathrm{A}^{\prime} \alpha \Delta \mathrm{J} \alpha$ derivative with these helices truncated. Consistent with the removal of $\mathrm{A}^{\prime} \alpha$ and $J \alpha$, this variant exhibited a $20 \%$ reduction in $[\theta]_{\text {MRW }}$ at $220 \mathrm{~nm}$ (Fig. 4f). Rather than a decrease, blue light elicited a small signal gain around $208 \mathrm{~nm}$ which we tentatively ascribe to flavin photoreduction, given that both the quinone and NSQ states strongly absorb in the far-UV region. By contrast, we did not observe any loss in $\alpha$-helical structure from which we concluded that the light-induced structural changes in AsLOV2 C450A:Q513D are likely caused by partial unfolding of the terminal $A^{\prime} \alpha$ and J $\alpha$ helices. Although the amplitude of the structural response is greatly reduced compared to wild type, it is striking that light-induced responses can be elicited in the absence of two strictly conserved active-site residues.

313

\section{Molecular bases of LOV signal transduction without the active-site glutamine.}

The above findings compellingly show that several LOV receptors transduce light signals in the absence of the active-site glutamine, long considered essential. To arrive at a molecular understanding, we solved the crystal structures of AsLOV2 wild-type and Q513L in the dark-adapted states to resolutions of $1.00 \AA$ and 0.90 $\AA$, respectively. Notably, both AsLOV2 variants formed crystals at the previously published solution conditions ${ }^{28}$ and adopted the same space group with closely similar cell dimensions (Suppl. Tables S1 and S2). To additionally acquire information on the light-adapted state, we pursued a freeze-trapping strategy. Dark-grown crystals were exposed to blue light and rapidly cryo-cooled, X-ray diffraction was recorded, and structures were refined to resolutions of $1.09 \AA$ (wild type) and $0.98 \AA$ (Q513L) (Suppl. Tables S1 and S2). Although the crystal lattice stands to influence any structural rearrangements, in the past light-induced conformational transitions could thus be resolved for several LOV receptors $8,19,28,55$, if likely at reduced amplitude and extent than in solution. Overall, the dark- and light-adapted states of AsLOV2 wild-type and Q513L exhibited closely similar structures with pairwise root mean-square displacement (rmsd) values of 0.33 to $0.36 \AA$ for the mainchain atoms of residues 404-546 (Suppl. Fig. S6). Differences among the four structures were subtle and concentrated on the chromophore-binding pocket and its surroundings (Fig. 5). Notably, these differences 
were consistent across several crystals, implying that they are genuinely tied to the $\mathrm{Q} 513 \mathrm{~L}$ exchange and illumination, respectively.

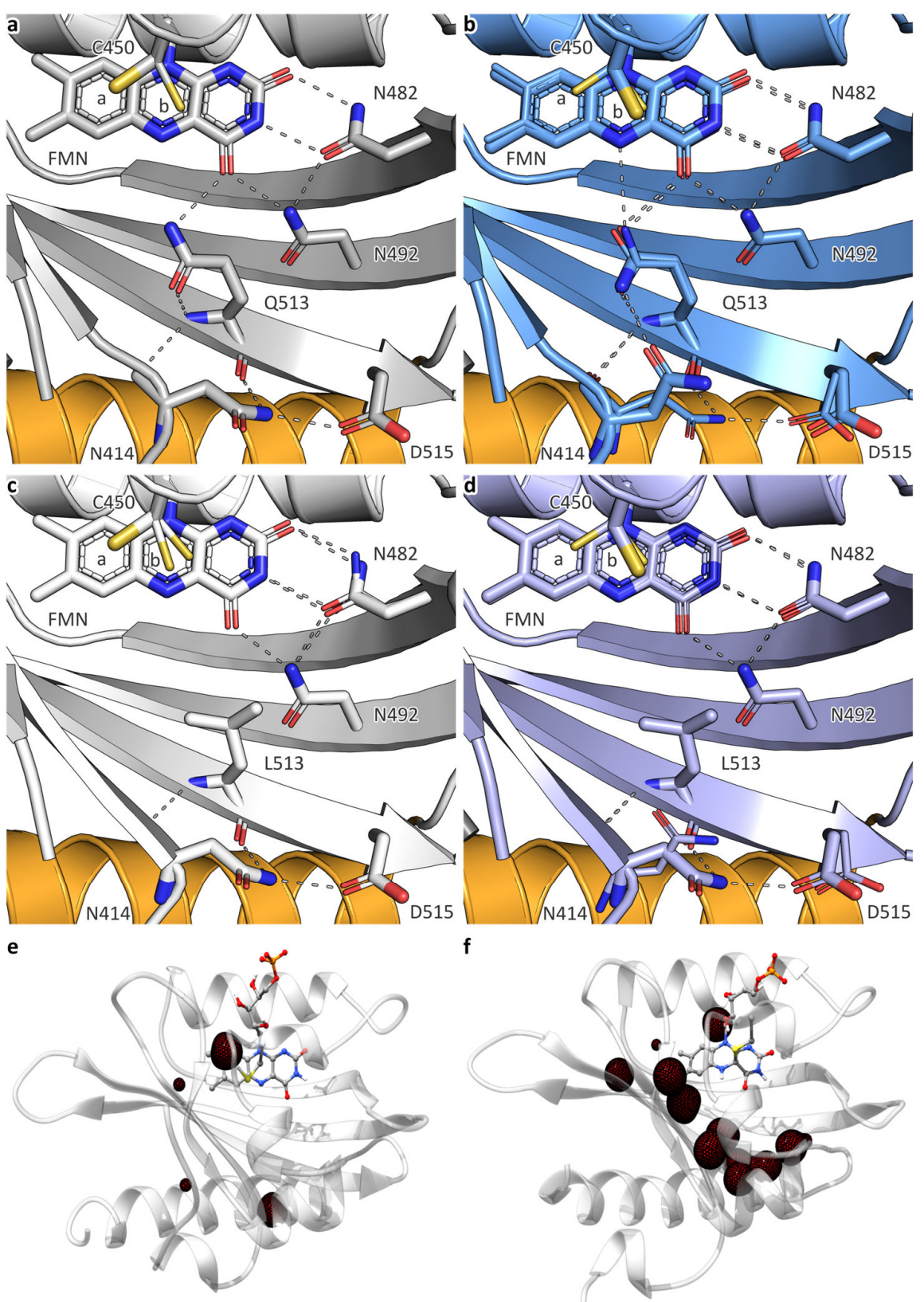

Fig. 5 - Structural analyses of AsLOV2 variants. a, Chromophore-binding pocket of wild-type AsLOV2 in its dark-adapted state as revealed by a $1.00 \AA$ crystal structure. b, Chromophore-binding pocket of wild-type AsLOV2 in its light-adapted state as revealed by a $1.09 \AA$ crystal structure. c, Chromophorebinding pocket of AsLOV2 Q513L in its dark-adapted state as revealed by a $0.90 \AA$ crystal structure. d, Chromophore-binding pocket of AsLOV2 Q513L in its light-adapted state as revealed by a $0.98 \AA$ crystal structure. For clarity, helices $C \alpha$ and $D \alpha$ are not shown in panels a-d. The J $\alpha$ helix is drawn in orange, and the flavin mononucleotide cofactor and key amino acids are highlighted in stick representation. Minor conformations of residues and the flavin nucleotide are drawn in narrower diameter. Dashed lines denote hydrogen bonds. e, Water density in the interior of dark-adapted AsLOV2 Q513L derived from a 300 ns classical molecular dynamics simulation. The red mesh denotes a density level of 0.3 water molecules per $\AA^{3}$. f, As panel e but for light-adapted AsLOV2 Q513L. 
The structure of dark-adapted AsLOV2 wild type (Fig. 5a) well agreed with a previous determination at $1.4 \AA$ (PDB entry $2 \mathrm{v} 0 \mathrm{u}$, mainchain rmsd $0.13 \AA \mathrm{C}^{28}$. As observed before, the active-site cysteine 450 adopted a major (80\%) conformation a, pointing away from the flavin C4a atom, and a minor (20\%) one b, oriented towards C4a (Suppl. Fig. S7). The flavin pteridin moiety was coordinated by the asparagines N482 and N492,

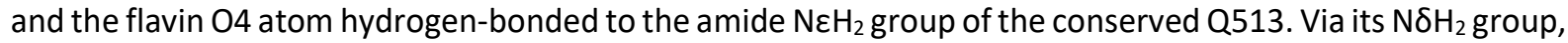
N414 at the start of strand A $\beta$ entered hydrogen bonds with the backbone carbonyl oxygen of Q513 and the carboxylate group of D515, situated at the tip of strand I $\beta$ and part of the conserved PAS DIT motif ${ }^{6}$. At the present high resolution, an alternate conformation could be resolved for the terminal turn of the Ja helix (residues 543-546), possibly reflecting the inherent equilibrium between folded and unfolded helical states 15,56 .

The light-adapted state of AsLOV2 wild type (Fig. 5b) exhibited a series of conformational differences consistent with a previous report at $1.7 \AA$ resolution (PDB entry $2 \mathrm{v} 0 \mathrm{w}$, mainchain rmsd $0.21 \AA$ ) ${ }^{28}$. Given the higher resolution achieved presently, additional structural transitions could be pinpointed as summarized below. The sidechain of $\mathrm{C} 450$ reoriented towards the flavin $\mathrm{C} 4 \mathrm{a}$, thus shifting the ratio of the conformations a and b to 40\%:60\% (Suppl. Fig. S7). As in other structures of photoactivated LOV receptors, e.g., 8,28 , little electron density for the cysteinyl-flavin thioadduct was observed, likely owing to X-ray radiolysis of the metastable thioether bond. Beyond the altered conformation of $\mathrm{C} 450$, the population of the light-adapted state was indicated by $\mathrm{a} \sim 6.9^{\circ}$ tilt of the isoalloxazine plane towards the cysteine (Suppl. Fig. S8) ${ }^{8,19}$. Based on earlier reports $8,19,28,55,57$, chemical reasoning and spectroscopic evidence ${ }^{18,20}$, the sidechain of the conserved glutamine Q513 was modelled to undergo a $180^{\circ}$ flip in response to enable hydrogen bonding between the amide $\mathrm{O} \varepsilon$ atom and the newly protonated flavin N5 position. Upon reorientation, the $\mathrm{Q} 513$ amide $\mathrm{N} \mathrm{H}_{2}$ group hydrogen-bonded with the backbone carbonyl O of N414. The asparagine 414 in turn rotated, thus breaking contact to D515 and enabling a new hydrogen bond between its amide $\mathrm{O} \delta$ atom and $\mathrm{N}^{2} \mathrm{H}_{2}$ of Q513 (Suppl. Fig. S7). Notably, the dark-adapted state conformations of both Q513 and N414 were retained as a minor population (20\%) in the light-adapted state, potentially due to incomplete photoactivation in the crystal. The reorientation of $\mathrm{N} 414$ correlated with a 0.4 A shift of its $\mathrm{C} \alpha$ atom, thereby prompting the entire $\mathrm{A}^{\prime} \alpha$ segment to dislodge and move away from Q513 (Suppl. Fig. S9). Crucially, the $A^{\prime} \alpha$ helix is interlocked with the C-terminal part of J $\alpha$ via the hydrophobic residues L408, 1411, 1539, A542, and L546. The displacement of $A^{\prime} \alpha$ thus went along with an outward movement of the last 1.5 helical turns of $J \alpha$, which could potentially promote its unfolding ${ }^{15}$. Support for this notion derives from the well-documented detrimental effect of the I539E substitution at the $A^{\prime} \alpha: J \alpha$ interface ${ }^{48}$ and from a recent study on circularly permuted AsLOV2 which pinpointed the J $\alpha \mathrm{C}$ terminus as pivotal for light-dependent signaling, whereas the $\mathrm{N}$-terminal part could be dispensed with ${ }^{32}$. In addition to the above differences, the light-adapted state also exhibited enhanced flexibility of the $A \beta-B \beta$ and $G \beta-H \beta$ loops, consistent with a global gain of mobility upon light absorption in AsLOV2 and other LOV domains ${ }^{15,58}$. 
In dark-adapted AsLOV2 Q513L (Fig. 5c), the flavin plane was displaced by around 0.4 A relative to the wild-type protein, arguably due to steric interactions between the flavin $\mathrm{O} 4$ and the $\mathrm{C} \delta 2$ methyl group of L513. Notably, no ordered water molecules entered the space vacated by the glutamine removal. The resultant loss of hydrogen bonds at the flavin $\mathrm{O} 4$ atom may account for the hypsochromic absorbance shift evidenced above across the different LOV receptors with replaced glutamine. C450 adopted the orientations a and b, pointed away and towards the flavin C4a atom, respectively, at a ratio of 70\%:30\% (Suppl. Fig. S7). The Q513L replacement notwithstanding, the crucial N414 residue assumed the conformation seen in darkness for the wild type, i. e. engaged in hydrogen bonds with D515 and the backbone carbonyl O of residue 513. Interestingly, the $Q 513 L$ dark state showed alternate conformations for the $A \beta-B \beta$ and $G \beta-H \beta$ loops, in case of the wild-type receptor only seen upon light exposure. Despite lacking the conserved glutamine, the AsLOV2 Q513L variant displayed structural responses in its light-adapted state structure remarkably similar to the wild type, in line with the above functional assays that invariably demonstrated qualitatively intact light responses after leucine introduction. Specifically, C450 adopted the conformations a and b at a 40\%:60\% ratio, and the flavin ring plane tilted towards the cysteine by around $4.6^{\circ}$. Strikingly, L513 did not exhibit any dark-light differences, implying that its sidechain is inert and not actively participating in signal relay. This notion is supported by the observation that most of the canonical amino acids with diverse sidechains supported productive light responses in the YF1 receptor (see Fig. 2b). Intriguingly, the crucial N414 assumed the light-adapted conformation to $40 \%$ extent; signals were evidently transduced from the flavin to this site even in the absence of the intermediary glutamine, if at reduced efficiency compared to wild-type AsLOV2. Rotation of the asparagine sidechain was accompanied by the same structural transitions evidenced in the wild-type receptor, most importantly an outward shift of the N414 C $\alpha$ atom and the complete A' $\alpha$ segment (Suppl. Fig. S9).

Collectively, the data reveal at high resolution how light stimuli propagate from the flavin to the LOV $\beta$-sheet interface and the terminal $A^{\prime} \alpha$ and J $\alpha$ helices, structural elements generally associated with downstream signal transduction across LOV domains ${ }^{15,26,27,36,59,60}$. Strikingly, the Q513L variant underwent the same qualitative responses as wild type which raises the question how signal relay to N414 and beyond can be rationalized in the absence of the glutamine? As candidate mechanisms, we principally considered electrostatic interactions through space and water-mediated rearrangement of hydrogen-bonding networks. To assess the validity of these proposals, we resorted to molecular simulations. Electrostatics calculations revealed that in wild-type AsLOV2 the light-induced formation of the cysteinyl-flavin thioadduct and accompanying flavin N5 protonation prompt changes in the electrostatic potential that are small in size, largely confined to the chromophore itself and not extending far in space (Suppl. Fig. S10). Highly similar electrostatic potentials resulted for the corresponding AsLOV2 Q513L structures, and we thus deem signal transduction through space via altered electrostatics unlikely. Although the light-state structures of AsLOV2 wild-type and Q513L did not exhibit ordered water molecules in the immediate vicinity of position 513, we hypothesized that water might transiently enter the chromophore-binding pocket and thus relay the N5 protonation 
change in the light-adapted state. This notion finds support in classical molecular dynamics (MD) simulations that indicate water penetration into the flavin binding pocket upon light exposure (Fig. 5e, f). Whereas in the simulations of dark-adapted AsLOV2 Q513L only two significant water clusters were observed inside the protein, the light-adapted state seemed to "soak" up water from the bulk solvent and displayed nine clusters in the protein interior. Closely similar results were obtained in simulations on AsLOV2 wild type (Suppl. Fig. 11a, b). This striking phenomenon can be rationalized by reduced rigidity of the protein backbone upon formation of the cysteinyl adduct (Suppl. Fig. 11c, d). The pairwise root mean square deviation between snapshots from the MD trajectory was below $1.8 \AA \AA$ for dark-adapted AsLOV2 Q513L but lay in the region of $2.4 \AA$ and higher for the light-adapted state. We note that these findings concur with the above-mentioned increase in general protein mobility evidenced in LOV receptors upon thioadduct formation ${ }^{15,58}$.

Signal transduction in natural glutamine-deficient LOV receptors.

Given that LOV signal transduction evidently does not strictly depend on the conserved glutamine, we wondered whether LOV-like receptors exist in nature that lack this residue. To address this question, we conducted sequence searches and identified around 350 putative LOV receptors, denoted LOV ${ }^{\triangle Q}$ in the following, that possess several residues highly conserved across LOV domains ${ }^{29}$ but lack the active-site glutamine (Fig. 6a and Suppl. Fig. S12). Interestingly, these receptors featured a range of other amino acids in lieu of the active-site glutamine, predominantly the hydrophobic amino acids leucine and isoleucine, but also polar residues as serine or threonine, and even histidine and cysteine. By contrast, large aromatic residues (phenylalanine, tyrosine, and tryptophan) were largely absent, as were proline and charged amino acids (Suppl. Fig. S13).

\section{a}

AsLOV2 AML79545.1 AML77488.1 WP_140774521.1 OYY45411.1

\section{AsLoV2} AML79545.1 AML77488.1 WP_140774521.1 OYY45411.1

435
401 GIDLATTLERIEKNFVITDPRLPDNPIIFASDSFLQLTEYSREEILGRNCRFLQGPETDRA
268 SKELKDALSTFQQTFLVADATKPDYPVLYASAGFFKMTGYAKEVIGRNCRFLQGAGTNPE
274 -SSLSIALNRIHQSFVLADPHLPDMPIVHASNLFLQLTGYSRDEVLGRNCRFLQGRDTDKA
28 EGIAARLPDLSRDGLAISDLREKGMALVYVRAFQDITGYSGDELIGRNCRFLQGSDRLQP
422 LRLQSSAMESSIDGILIADARITDMPLIYVNPAFERISGYAAEVLGKNCRFLGDDRAQP
462 TVRKIRDAIDNQTEVTVQLINYTKSGKKFWNLFHLQPMRDQKGDVQYFIGVLDGTEHVRD
329 DISKIREALAERRSYCGRILNYKKDGTPFWLLTIAPIKDETGNTLKFIGMLVEVSKHTEG
334 AVAQIRQSIVAEHCCTVRILNYRKDNSSFWNLLHTAPVRNASGKVAFYVGVHLDVTGIDEE
89 EIQSIREAIARREDVAVTLKNYRKDGSFWNELRLSPMSIAGGEPTHYIGLMRDVTASRVA
483 GLEIIREAFREQRACTVLLRNYRKTGELYWNDLTLSPVRNNGGDITHYIGVCNDVTERTRV Cᄂ IR AL

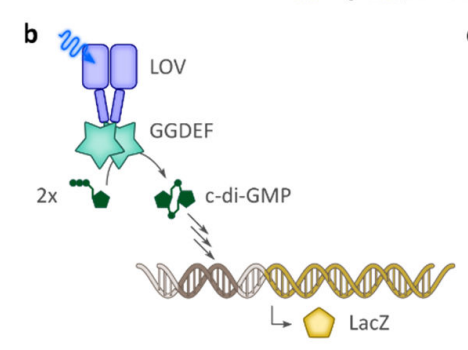

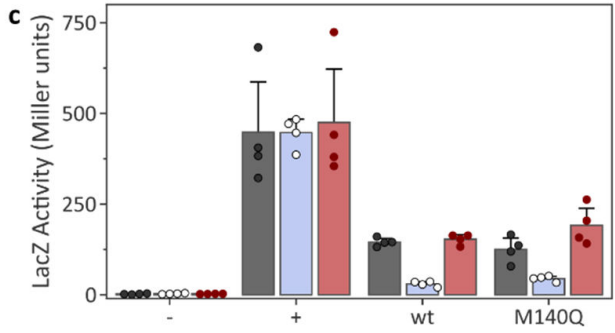


Fig. 6 - Naturally occurring, glutamine-deficient LOV ${ }^{\Delta Q}$ receptors. a, Sequence searches identify around 350 receptors that have homology to bona fide LOV receptors but lack the conserved active-site glutamine. The multiple sequence alignment shows AsLOV2 as a reference and four selected glutaminedeficient receptors. The sequence logo below the alignment was calculated for the entire set of glutamine-deficient LOV receptors (see Suppl. Fig. S12). Coloring, shading, and arrows as in Fig. 1, with the position of the conserved glutamine residue indicated by a red arrow. $\mathbf{b}$, Activity and light response of the LOV ${ }^{\triangle Q}$ _GGDEF fragment of WP_140774521.1 were assessed in an E. coli reporter strain harboring a $d g c E$ knockout and a translational fusion between the cyclic-di-GMP-controlled csgB and lacZ. Lightdependent diguanylate cyclase activity can hence be assessed by measuring $\beta$-galactosidase levels. $\mathbf{c}$, Bacteria expressing the wild-type LOV ${ }^{\triangle Q}{ }_{-G G D E F}$ receptor or the $M 140 \mathrm{Q}$ variant were cultivated in darkness (black dots and grey bars), under blue light (white dots and blue bars), or under red light (red dots and bars). '-' refers to an empty-vector negative control, and ' + ' denotes a strain expressing the major diguanylate cyclase DgcE that served as the positive control. $\beta$-galactosidase activity is reported in Miller units and represents mean \pm s.d. of four biologically independent replicates. The experiment was repeated twice with similar outcome.

The sheer existence of LOV ${ }^{\Delta Q}$ proteins in nature raises the tantalizing prospect that they can truly serve as blue-light receptors. To principally address this possibility, we selected for further analysis a LOV ${ }^{\triangle Q}$-GGDEFEAL receptor from the proteobacterium Mesorhizobium loti which features a methionine at position 140 instead of the conserved glutamine (Genbank entry WP_140774521.1, see Fig. 6a). GGDEF and EAL domains antagonistically synthesize and degrade, respectively, the ubiquitous bacterial second messenger cyclic-di$\left(3^{\prime}-5^{\prime}\right)$-guanosine monophosphate (c-di-GMP) ${ }^{61}$. To assess potential light responses, we expressed the Cterminally truncated LOV ${ }^{\Delta Q_{-}}$GGDEF receptor in the $E$. coli reporter strain KN78 which lacks the major diguanylate cyclase DgcE and carries a translational fusion between the c-di-GMP-controlled csgB locus and $\beta$-galactosidase $^{62}$ (Fig. 6b). Bacteria were cultivated in darkness, under blue light, or under red light, and $\beta$-galactosidase activity was determined. As a positive control, a strain expressing DgcE exhibited constitutively high activity of around 450 Miller units (M.u.), irrespective of illumination (Fig. 6c). The KN78 strain carrying an empty plasmid served as a negative control and showed low activity of around 2 M.u., again independent of light. LOV ${ }^{\triangle Q}$-GGDEF expression resulted in 145 M.u. in darkness but only 30 M.u. under blue light. Conversely, red light had no effect on the detectable activity. Replacement of M140 by glutamine yielded activity levels and light responses similar those of the wild-type protein. Taken together, the results suggest that the $M$. loti LOV $^{\triangle Q_{-} G G D E F}$ acts as a blue-light-repressed diguanylate cyclase despite lacking the conserved glutamine residue.

\section{Discussion}

\section{Mechanism of signal transduction sans glutamine.}

Following the description of light-oxygen-voltage receptors as blue-light-receptive flavoproteins ${ }^{1}$, optical and nuclear magnetic resonance spectroscopy identified formation of the cysteinyl-flavin adduct in the signaling state ${ }^{10,18}$. Owing to a hybridization change of the flavin C4a atom from $s p^{2}$ to $s p^{3}$ in the adduct, the adjacent 
N5 atom is protonated and thus converted from a hydrogen-bond acceptor in the dark-adapted state to a donor in the signaling state (Suppl. Fig. S14). N5 protonation is an essential step in signal transduction as not least evidenced by reconstitution of LOV receptors with 5-deaza-FMN ${ }^{63}$. Despite retaining the ability to form the thioadduct under blue light, these receptors are incapable of downstream signaling responses, arguably due to a lack of hydrogen bonding at the C5 position. Further support for the pivotal role of N5 protonation derives from cysteine-deficient LOV receptors that undergo photoreduction to the NSQ state which is protonated at N5 and thus elicits intact signaling responses ${ }^{14}$. Three-dimensional structures of phototropin LOV domains early on pinpointed the conserved glutamine residue close-by the flavin chromophore and in hydrogen-bonding distance to the $\mathrm{O} 4$ and N5 atoms ${ }^{7,8,19}$. Supported by spectroscopic evidence ${ }^{18,20,64}$, the glutamine is generally held to rotate its sidechain upon N5 protonation to satisfy hydrogen bonding ${ }^{8,19}$. Possibly, this rotation is aided by transient rearrangements of two conserved asparagines (residues N482 and N492 in AsLOV2, see Fig. 5) that coordinate the flavin nucleotide chromophore ${ }^{24,63}$. Reorientation of the glutamine residue in turn provokes a cascade of hydrogen-bonding and structural changes, as for instance revealed in the past ${ }^{28}$ and present structures of light-adapted AsLOV2 (see Fig. 5). Photochemical reactions within the flavin chromophore, i.e. thioadduct formation or reduction to the NSQ state ${ }^{14}$, are thus coupled to the protein scaffold, in particular the LOV $\beta$ sheet and elements contacting it, e.g., $\mathrm{N}$ - and C-terminal extensions to the core domain. In AsLOV2 specifically, asparagine 414 responds with a sidechain flip, accompanied by a shift of the protein backbone. Signals are thus channeled to the $A^{\prime} \alpha$ and J $\alpha$ helices and likely drive their lightdependent unfolding.

Irrespective of the strong conservation of the glutamine and its central involvement in canonical LOV signal transduction, its removal unexpectedly does not abolish light-dependent signaling responses. Intriguingly, this effect spans LOV receptors of distant phylogenetic origin and with disparate associated output modules (see Figs. 2-5), that invariably retained intact responses upon replacement of the glutamine, if to different and often reduced quantitative extent. In line with these observations, two recent reports revealed that the LOV domains from Vaucheria frigida aureochrome 1 and $A$. thaliana ZTL also elicited intact downstream responses after replacement of the glutamine by leucine or other residues ${ }^{30,31}$. Taken together, we propose that the conserved glutamine, long considered essential for LOV signal transduction, is in fact generally dispensable. This view is corroborated by the existence of hundreds of glutamine-deficient LOV ${ }^{\Delta \mathrm{Q}}$ proteins in nature (see Fig. 6a, Suppl. Fig. S12 and ${ }^{30}$ ), which presumably serve as blue-light receptors, as we presently demonstrate for a proteobacterial LOV ${ }^{\Delta Q_{-} G G D E F}$ protein (see Fig. 6b).

Our functional and structural data suggest a potential mechanism for signal transduction in glutaminedeficient LOV receptors. The observation that most amino acids can stand in for the glutamine and support intact signal transduction (see Figs. 2 and 5) immediately argues against a direct involvement of the sidechain of these residues. Strikingly, the crystal structures of AsLOV2 wild-type and Q513L revealed highly similar light-induced conformational changes that culminated in reorientation and altered hydrogen bonding of N414 and translocation of the $A^{\prime} \alpha$ segment. The problem of signal transduction in glutamine-deficient LOV 
receptors thus reduces to the question of how signals are relayed across $10 \AA$ from the newly protonated N5 atom to the LOV $\beta$ sheet, and specifically to N414 in AsLOV2. In the following, we principally consider and discuss in turn as potential mechanisms i. steric rearrangements near the chromophore; ii. altered electrostatics in the thioadduct state; and iii. water-mediated hydrogen-bonding changes. First, as recently proposed for $A$. thaliana $\mathrm{ZTL}^{30}$, steric rearrangements upon adduct formation, i.e. bond strain, $s p^{2} \rightarrow s p^{3}$ hybridization change of the $\mathrm{C} 4 \mathrm{a}$ atom, and tilting of the isoalloxazine heterocyclic system ${ }^{8,19}$, might underpin signal propagation. However, the light-state Q513L structure did not reveal substantial conformational changes of residues immediately next to the flavin. Moreover, as previously demonstrated ${ }^{14}$, cysteine-deficient LOV receptors can elicit canonical signaling responses when photoreduced to their NSQ state which is protonated at the N5 position like the thioadduct but experiences different steric constraints. Taken together, we thus regard steric effects as an unlikely general mechanism for signal propagation in glutamine-deficient receptors but note that for specific LOV proteins they plausibly play a crucial role ${ }^{30}$. Second, formation of the thioadduct evidently modifies the electronic structure of the flavin and gives rise to an altered electrostatic potential. However, molecular simulations revealed (see Fig. 5) that such changes in electrostatics are comparatively small and of short reach. We hence deem it unlikely that electrostatic interactions transmitted through space are causative for signal transduction. Rather, we favor the third option of water-mediated hydrogenbonding rearrangements, as illustrated in Fig. 7.

a

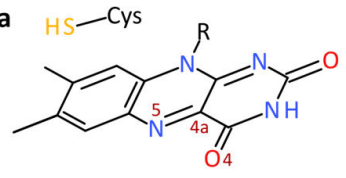
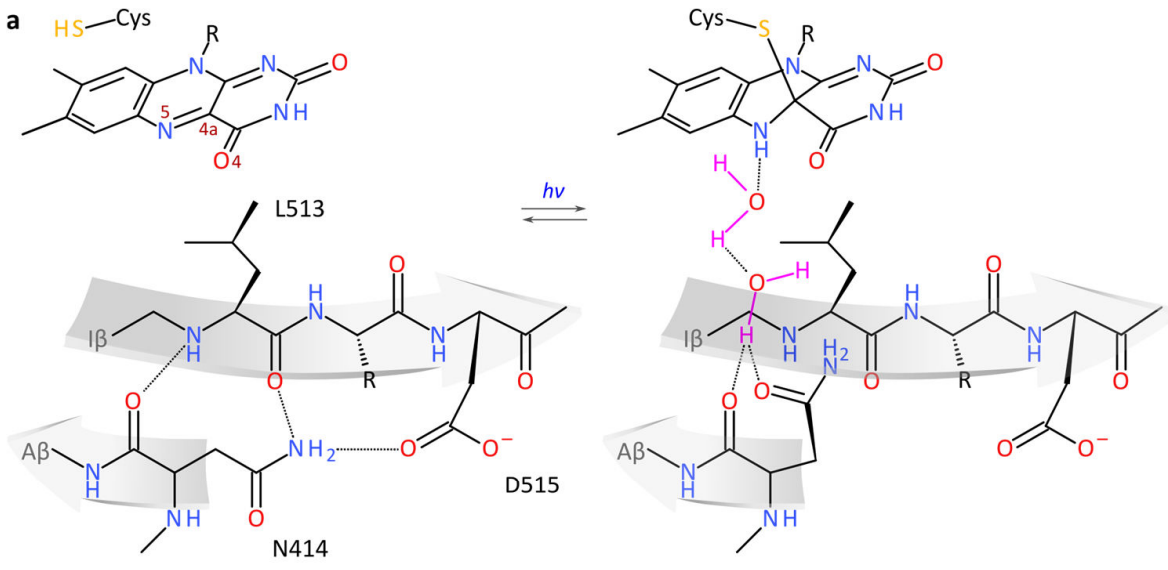

b

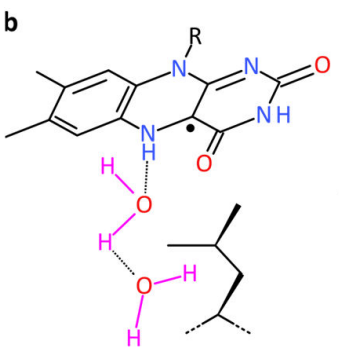

ancestral

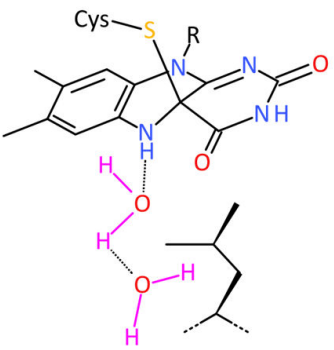

glutamine-devoid

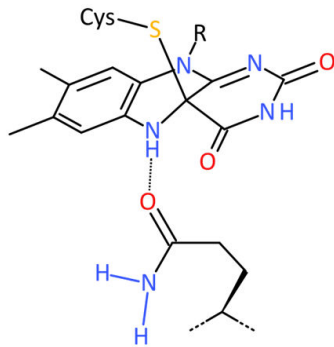

recent

Fig. 7 - Signal transduction in light-oxygen-voltage (LOV) receptors lacking the conserved glutamine, exemplified for the $A$. sativa phototropin 1 LOV2 domain. a, Lewis formulae show the flavin nucleotide chromophore and surrounding residues of the glutamine-deficient leucine variant in the dark-adapted (left) and light-adapted states (right). As revealed by X-ray crystallography (see Fig. 5), qualitatively 
similar structural responses to light-induced N5 protonation (see Fig. 1) are observed in both the ab-

533

534

535

536

537

538

539

540

541

542

543

544

545

546

547

548

549

550

551

552

553

554

555

556

557

558

559

560

561

562

563

564

565

566

567

568

569 sence of the conserved glutamine Q513 and in its presence (see Suppl. Fig. S14). Without the glutamine, water molecules might transiently enter the chromophore-binding pocket, thereby stand in for the glutamine, and relay the signal as changes in hydrogen bonding to the $I \beta$ and $A \beta$ strands of the central $\beta$ pleated sheet (involving residues N414 and 513). Notably, signals are thus also propagated to the LOV C terminus (D515) that is frequently engaged in signal transduction and often exhibits a conserved DIT motif ${ }^{6} . \mathbf{b}$, The observation that LOV receptors can transduce signals without either or both of their strictly conserved cysteine and glutamine residues suggests a potential origin from redox-active flavoproteins ${ }^{14}$. LOV signal transduction in a primordial LOV ancestor lacking the Cys and GIn residues would have relied on flavin photoreduction to the NSQ radical and on water mediation. Both the Cys and GIn residues would be secondary acquisitions that minimize side reactions (Cys); enhance the fidelity of signal transduction (Cys and GIn); bathochromically shift the action spectrum (Gln); accelerate the dark recovery and thereby benefit temporal resolution (Cys and Gln); and render the signaling state less susceptible to the cellular environment (Cys). Note that we have no evidence in which sequential order the Gln and Cys residues may have been acquired.

We propose that water molecules transiently enter the flavin-binding pocket, occupy the space vacated by glutamine removal, and form hydrogen bonds to the protonated flavin N5 and N414. Water would thus substitute for the glutamine side chain of canonical LOV receptors and relay hydrogen-bonding changes originating at the chromophore to the LOV $\beta$ sheet, and N414 in case of AsLOV2. We note that neither the dark-adapted nor the light-adapted structures of AsLOV2 Q513L revealed direct evidence for ordered water molecules near the flavin N5 atom. However, support for our model derives from MD simulations suggesting that water dynamically enters this region of the light-adapted receptor. Moreover, the model would explain why, as one of only few amino acids, proline cannot functionally substitute for glutamine, despite leaving chromophore binding and LOV photochemistry intact. In the imino acid proline, the $C_{\gamma}$ and $C \delta$ methylene groups of the sidechain loop back onto the amide nitrogen atom, thus sterically interfering with the proposed water-mediated hydrogen bonding. Alternatively, we cannot however rule out that proline fails to convey light signals because of its restricted conformational freedom or its lack of an amide proton. Lastly, the proposed mechanism would rationalize the near-identical conformational changes elicited by light in both AsLOV2 wild-type and Q513L. Regardless of the presence of the glutamine, light signals would initially be converted into altered flavin N5 protonation and a subsequent hydrogen-bonding cascade that propagates to N414 at the LOV $\beta$-sheet interface ${ }^{28}$. Concomitant with formation of new hydrogen bonds, N414 would break or weaken the hydrogen bonds formed in darkness between its backbone oxygen and the amide proton of residue 513 , and between its ${\mathrm{N} \delta \mathrm{H}_{2}}_{2}$ amide and the sidechain of $\mathrm{D} 515$, respectively. The resultant weakening of the LOV $\beta$ sheet would then transmit to the $A^{\prime} \alpha$ and Ja helices that interact with the outer face of the sheet.

Although residue N414 is not strictly conserved (see, e.g., Fig. 1 and Suppl. Fig. S1), the proposed mode of signal transmission principally extends to other LOV receptors. Even in the absence of a polar residue at the position equivalent to $\mathrm{N} 414$, hydrogen-bond rearrangements could still be relayed to the $\beta$ sheet and 
beyond, as for instance evidenced in Neurospora crassa Vivid ${ }^{22,57}$. Across several LOV receptors, the outer $\beta$ sheet face and the adjacent DIT motif ${ }^{6}$ recurringly take center stage in signal transduction ${ }^{15,26,27,37,60,65}$. Once relayed there, signals are then channeled into disparate structural responses in individual LOV receptors, including order-disorder transitions, association reactions, and quaternary structural transitions ${ }^{5}$. It is worth noting that our mechanistic proposal is not in contradiction to common models advanced for signal transition in the presence of the glutamine, for instance a recent suggestion that two conserved asparagine residues crucially contribute ${ }^{24}$. Rather, by principally rationalizing how signal transduction occurs in the absence of the glutamine, our model reinforces the central roles of N5 protonation and hydrogen bonding in LOV signal transduction, which likely also applies to receptors with intact glutamine.

\section{LOV passes the QC}

Our data demonstrate that LOV receptors can evidently transduce light signals without the conserved glutamine. As qualitatively intact light responses are evoked upon glutamine replacement across all systems tested, we consider signaling in the absence of the glutamine a general and inherent, yet dormant trait of LOV receptors. This view is borne out by the existence of numerous glutamine-deficient LOV ${ }^{\Delta \mathrm{Q}}$ receptors in nature that could potentially serve as bona fide blue-light receptors. In a similar vein, we previously showed that LOV ${ }^{\Delta C}$ receptors devoid of the conserved cysteine exist in nature and can elicit productive light responses owing to photoreduction to the NSQ state which is protonated at the flavin N5 atom ${ }^{14}$. We show presently that the paradigm AsLOV2 domain perplexingly retains signaling capability, if at greatly attenuated efficiency, even when both the conserved cysteine and glutamine are replaced. Building on our earlier proposal ${ }^{14}$, these observations jointly raise the prospect that LOV receptors arose during evolution from originally light-inert flavoproteins, e.g., enzymes involved in redox processes (Fig. 7b). The question then begs, if signal transduction can take place in the absence of the cysteine and glutamine, why are these residues so prevalent in recent LOV receptors. Our data provide clues as to the potential driving forces underlying the strong glutamine conservation. First, introduction of the glutamine generally enhances the fidelity and degree of the light response. Second, glutamine induces a bathochromic absorbance shift of approximately $10 \mathrm{~nm}$, thus expanding light sensitivity to longer wavelengths. Third, glutamine accelerates the base-catalyzed dark recovery reaction ${ }^{64}$, thus enhancing temporal resolution of light-dependent physiological responses. Similarly, the cysteine may have prevailed as its introduction minimizes side reactions (fluorescence and photosensitizing), desensitizes the light-adapted signaling state against environmental influences (e.g., partial oxygen pressure and redox conditions), and enhances the fidelity of the signaling response ${ }^{14}$.

Beyond implications for the potential origin of LOV receptors, our data directly pertain to applications in optogenetics and biotechnology. First, replacement of the conserved glutamine residue generally decelerated the dark recovery kinetics but preserved signaling responses to substantial extent. Targeted modification of the glutamine residue thus provides a so-far little explored avenue towards modulating these kinetics 
and thus the effective light sensitivity at photostationary state (see Suppl. Fig. S3) ${ }^{41,42}$. In a similar vein, glutamine substitution may serve to deliberately attenuate the light response as demanded by application. Second, substitutions of either the conserved cysteine or glutamine residues have often been used as presumably light-insensitive, unresponsive negative controls. Our data however illustrate that even when these residues are replaced, LOV receptors can principally transduce light signals, although likely with reduced amplitude. These considerations transcend the optogenetic deployment of LOV receptors and also concern the widespread applications of cysteine-deficient (and often additionally glutamine-deficient ${ }^{66}$ ) LOV modules as fluorescent proteins ${ }^{67,68}$ and photosensitizers for molecular oxygen ${ }^{53}$.

\section{Methods}

\section{Molecular biology}

YF1 variants with residue Q123 replaced were constructed in the background of the pDusk-DsRed and pDawn-DsRed reporter plasmids ${ }^{35}$, or the expression plasmid pET-41a-YF1 ${ }^{36}$ according to the QuikChange protocol (Agilent Technologies). The gene of the cognate response regulator BjFixJ from Bradyrhizobium diazoefficiens, formerly designated B. japonicum, was amplified from an earlier expression construct ${ }^{37}$, subcloned onto the pET-19b vector (Novagen) and thus furnished with an N-terminal His ${ }_{6}-\mathrm{SUMO}$ tag. Substitutions of residue Q347 in the NmPAL receptor were performed via QuikChange in either the pCDF-PALopt reporter plasmid or the pET-28c-PALopt expression plasmid ${ }^{26}$. For the expression of AsLOV2, a gene encoding residues 404-546 of $A$. sativa phototropin 1 (Uniprot 049003) was synthesized with an N-terminal GEF extension ${ }^{15,28}$ and codon usage adapted to $E$. coli (GeneArt), and was cloned into the pET-19b vector. Notably, AsLOV2 was thus equipped with an $\mathrm{N}$-terminal $\mathrm{His}_{6}$-SUMO tag and its expression put under the control of a T7-lacO promoter. Replacements of the active-site residues Q513 and C450 were generated by QuikChange. Deletions of the $\mathrm{N}$ - and C-terminal $\mathrm{A}^{\prime} \alpha$ and J $\alpha$ helices were prepared by PCR amplification and blunt-end ligation of the vector; the resultant truncated AsLOV2 variant comprised residues 411-517. The gene encoding residues 1-326 of the glutamine-deficient LOV-GGDEF receptor (ANN58260.1/WP_140774521.1) was amplified by PCR from genomic DNA of the proteobacterium Mesorhizobium loti NZP2037 (purchased from Deutsche Sammlung für Mikroorganismen und Zellkulturen, DSMZ no. 2627) and cloned into the pQE-30 vector (Qiagen) via Gibson cloning ${ }^{69}$. Residue replacements were prepared by QuikChange. All oligonucleotide primers were purchased from Integrated DNA Technologies. All constructs were verified by Sanger sequencing (Microsynth AG, Göttingen).

\section{Protein expression and purification}

Protein expression and purification were carried out as previously described for YF1 ${ }^{36}$ and NmPAL ${ }^{26}$. To express and purify the response regulator BjFixJ, the above pET-19b BjFixJ expression plasmid was transformed into E. coli $\mathrm{BL} 21 \mathrm{CmpX13}$ cells ${ }^{70}$. Bacteria were grown at $37^{\circ} \mathrm{C}$ in Luria broth (LB) medium to an optical 
640

641

642

643

644

645

646

647

648

649

650

651

652

653

654

655

656

657

658

659

660

661

662

663

664

665

666

667

668

669

670

671

672

673

674

675

density at $600 \mathrm{~nm}\left(O D_{600}\right)$ of around $0.6-0.8$, at which point the temperature was lowered to $16^{\circ} \mathrm{C}$ and expression induced by addition of $1 \mathrm{mM}$ isopropyl $\beta$-D-1-thiogalactopyranoside (IPTG). Following incubation overnight at $16^{\circ} \mathrm{C}$, cells were lysed by sonication, and the supernatant was cleared by centrifugation and purified by $\mathrm{Ni}^{2+}$ immobilized metal ion affinity chromatography (IMAC). The His 6 -SUMO tag was cleaved off by the SUMO protease Senp2, followed by a second IMAC purification. BjFixJ protein was dialyzed into storage buffer [20 mM tris(hydroxymethyl)aminomethane (Tris)/ $\mathrm{HCl} \mathrm{pH} \mathrm{8.0,} 250 \mathrm{mM} \mathrm{NaCl}, 10 \%$ (w/v) glycerol], and the concentration was determined using an extinction coefficient of $4860 \mathrm{M}^{-1} \mathrm{~cm}^{-1}$ at $280 \mathrm{~nm}^{37}$.

For production of AsLOV2 variants, the pET-19b expression plasmid (see above) was transformed into E. coli BL21 CmpX13 or LOBSTR cells ${ }^{71}$. Protein expression was induced by addition of $1 \mathrm{mM} \mathrm{IPTG}$ and conducted at $16^{\circ} \mathrm{C}$ overnight. When using the $\mathrm{CmpX13}$ strain, the medium was supplemented with $50 \mu \mathrm{M}$ riboflavin. The cleared bacterial cell lysate was purified by $\mathrm{Co}^{2+}$ IMAC, Senp2 cleavage of the His 6 -SUMO tag and a second IMAC step, as described for BjFixJ. Depending on purity, AsLOV2 variants were further purified by anion-exchange chromatography. Purified protein was dialyzed into storage buffer $[20 \mathrm{mM}$ Tris/ $\mathrm{HCl}$ pH 7.4, $20 \mathrm{mM} \mathrm{NaCl}, 20 \%$ (v/v) glycerol], and its concentration was determined spectroscopically using an extinction coefficient of $13,800 \mathrm{M}^{-1} \mathrm{~cm}^{-1}$ for the flavin absorption maximum around $447 \mathrm{~nm}^{10}$.

\section{Spectroscopic analyses}

UV/vis absorbance spectra were recorded on an Agilent 8435 diode-array spectrophotometer at $22^{\circ} \mathrm{C}$, as controlled by an Agilent 89090A Peltier thermostat. Absorbance spectra were acquired for the dark-adapted LOV receptors and after saturating illumination with a 455-nm light-emitting diode (LED) ( $30 \mathrm{~mW} \mathrm{~cm}^{-2}$ ). Throughout the study, all light intensities were determined with a power meter (model 842-PE, Newport) and a silicon photodetector (model 918D-UV-OD3, Newport). The recovery to the dark-adapted state was monitored by recording spectra over time. The resultant kinetics were corrected for baseline drift and evaluated by nonlinear least-squares fitting to exponential functions using the Fit-o-mat software ${ }^{72}$. Absorbance spectroscopy on YF1 variants was conducted at $37^{\circ} \mathrm{C}$ in $20 \mathrm{mM}$ Tris/ $\mathrm{HCl} \mathrm{pH} 8.0,20 \mathrm{mM} \mathrm{NaCl}$; to accelerate the recovery in the Q123L variant, up to $1 \mathrm{M}$ imidazole was added ${ }^{13}$, and the resulting rate constants for dark recovery were extrapolated to $0 \mathrm{M}$ imidazole. UV/vis-spectroscopic analysis of NmPAL was performed in 12 mM 4-(2-hydroxyethyl)-1-piperazineethanesulfonic acid (HEPES)/HCl pH 7.7, $135 \mathrm{mM} \mathrm{KCl}, 10 \mathrm{mM} \mathrm{NaCl}, 1 \mathrm{mM}$ $\mathrm{MgCl}_{2}, 10 \%$ (v/v) glycerol ${ }^{26}$. AsLOV2 variants were analyzed in $10 \mathrm{mM}$ sodium phosphate pH 7.5, $10 \mathrm{mM}$ $\mathrm{NaCl}$; to aid solubility, for the Q513D variant $20 \%(\mathrm{v} / \mathrm{v})$ glycerol was added. To promote photoreduction in the cysteine-devoid AsLOV2 C450A variant, $1 \mathrm{mM}$ tris(2-carboxyethyl)phosphine (TCEP) was added.

Secondary structure and light-induced changes were assessed by circular dichroism (CD) spectroscopy on a JASCO J710 spectrophotometer equipped with a PTC-348WI Peltier element. CD spectra were recorded at $22^{\circ} \mathrm{C}$ in a 1-mm cuvette for the dark-adapted state and following saturating blue-light illumination for the light-adapted state. All spectra were corrected by blank spectra and represent the average of at least 4 scans. In case of the faster-recovering AsLOV2 variants, blue light was applied before each scan. Buffers were as 
above except for $\mathrm{NmPAL}$ where $12 \mathrm{mM}$ HEPES/HCl pH 7.7, $135 \mathrm{mM} \mathrm{KCl}, 10 \mathrm{mM} \mathrm{NaCl}, 1 \mathrm{mM} \mathrm{MgCl}$, 10\% (v/v) glycerol was used instead. In case of the AsLOV2 variants, the return to the dark-adapted state after bluelight exposure was monitored over time at a wavelength of $(208 \pm 5) \mathrm{nm}$ and evaluated by fitting to exponential functions using Fit-o-mat ${ }^{72}$.

\section{YF1 functional assays}

The net kinase activity of YF1 variants and its dependence on blue light were assessed in the pDusk-DsRed reporter setup ${ }^{35,73}$. To this end, pDusk-DsRed plasmids harboring different YF1 variants were transformed into E. coli $\mathrm{CmpX13}$. Individual wells of a 96-deep-well microtiter plate (P-DW-11-C-S, Corning, New York) containing $400 \mu \mathrm{L} \mathrm{LB}$ supplemented with $50 \mathrm{gg} \mathrm{mL}^{-1}$ kanamycin were inoculated with a given YF1 variant. Plates were sealed with a gas-permeable film (BF-410400-S, Corning) and incubated for $16 \mathrm{~h}$ at $37^{\circ} \mathrm{C}$ and 700 $\mathrm{rpm}$ in either darkness or under constant blue light $\left(470 \mathrm{~nm}, 100 \mu \mathrm{W} \mathrm{cm}{ }^{-2}\right)$. Following incubation, $O D_{600}$ and the fluorescence of the DsRed Express2 reporter ${ }^{74}$ were measured with a Tecan Infinite M200 PRO plate reader (Tecan Group Ltd. Männedorf, Switzerland). For the fluorescence measurements, the excitation wavelength was $(554 \pm 9) \mathrm{nm}$ and that of the emission $(591 \pm 20) \mathrm{nm}$. Fluorescence data were divided by $O D_{600}$ and normalized to the value for YF1 under dark conditions. Data represent the mean \pm s.d. of three biologically independent samples. The response to trains of blue-light pulses was assessed for pDawn-DsRed systems harboring different YF1 variants as previously described ${ }^{43}$. Briefly, bacterial cultures were grown in sealed, black-walled 96-well microtiter plates (Greiner BioOne, Frickenhausen, Germany) for $16 \mathrm{~h}$ at $37^{\circ} \mathrm{C}$ and $600 \mathrm{rpm}$. The transparent bottom of the plates allowed illumination from below with a programmable matrix of light-emitting diodes. Following incubation, $O D_{600}$ and $D s$ Red fluorescence were measured and evaluated as above.

Activity and light response of purified YF1 variants were characterized in a coupled assay that reports on the phosphorylation-induced binding of BjFixJ to a fluorescently labeled, double-stranded DNA (dsDNA). To this end, a dsDNA substrate with the sequence 5'-GAG CGA TAT CTT AAG GGG GGT GCC TTA CGT AGA ACC C-3' and labeled at its $5^{\prime}$ end with (5-and-6)-carboxytetramethylrhodamine (TAMRA) was prepared as described before ${ }^{14}$. The underlined portion of the sequence corresponds to the BjFixK2 operator site that $B j F i x J$ binds to ${ }^{45}$. To assess light-dependent catalytic activity, $2.5 \mu \mathrm{M}$ of each YF1 variant in its dark-adapted state were incubated at $25^{\circ} \mathrm{C}$ with $1.25 \mu \mathrm{M}$ BjFixK2 dsDNA substrate and $25 \mu \mathrm{M}$ BjFixJ in buffer containing $10 \mathrm{mM}$ HEPES/HCl pH 7.6, $80 \mathrm{mM} \mathrm{KCl}, 2.5 \mathrm{mM} \mathrm{MgCl}_{2}, 0.1 \mathrm{mM}$ ethylenediaminetetraacetic acid (EDTA), $111 \mu \mathrm{g} \mathrm{mL}^{-1}$ bovine serum albumin (BSA), 10\% (v/v) glycerol, $4 \%(\mathrm{v} / \mathrm{v})$ ethylene glycol and $20 \mathrm{mM}$ TCEP. The solution was transferred to a black 96-well microtiter plate (FluoroNunc). Upon starting the reaction by addition of $1 \mathrm{mM}$ ATP, the kinetics were followed by measuring TAMRA fluorescence anisotropy with a multi-mode microplate reader (CLARIOstar, BMG Labtech) over $30 \mathrm{~min}$. Fluorescence was recorded at excitation and emission wavelengths of $(540 \pm 10) \mathrm{nm}$ and $(590 \pm 10) \mathrm{nm}$, respectively, and using a 566 -nm long-pass beam splitter. After 
$30 \mathrm{~min}$, the microtiter plate was ejected, the samples illuminated for $30 \mathrm{~s}$ with a $470-\mathrm{nm}$ LED $\left(30 \mathrm{~mW} \mathrm{~cm}^{-2}\right)$, and the measurement continued for another $12 \mathrm{~min}$.

\section{NmPAL functional assays}

The light-dependent binding of NmPAL variants to their RNA target was assessed in a bacterial reporter-gene system ${ }^{26}$. Briefly, E. coli $\mathrm{CmpX13}$ cells ${ }^{70}$ were transformed with the arabinose-inducible pCDF-PALopt expression and the pET-28c-DsRed-SP reporter plasmids ${ }^{26}$. Notably, the reporter plasmid contains the NmPAL aptamer 04.17 upstream of the Shine-Dalgarno (SD) sequence of the DsRed gene; NmPAL binding to this site thus reduces reporter expression at the mRNA level. Bacterial starter cultures were grown at $37^{\circ} \mathrm{C}$ overnight, transferred to individual wells of a 96-deep-well microtiter plate, and diluted to an $O D_{600}$ of 0.03 in $700 \mu \mathrm{L} \mathrm{LB}$ medium supplemented with $4 \mathrm{mM}$ arabinose, $50 \mathrm{\mu g} \mathrm{mL}^{-1}$ kanamycin, and $100 \mathrm{mg} \mathrm{mL}^{-1}$ streptomycin. Following $2 \mathrm{~h}$ incubation at $37^{\circ} \mathrm{C}$ and $600 \mathrm{rpm}$, cultures were supplemented with $1 \mathrm{mM}$ IPTG to induce DsRed expression. Cultures were then split into two samples which were incubated for $16 \mathrm{~h}$ at $29^{\circ} \mathrm{C}$ in darkness or under blue light $\left(470 \mathrm{~nm}, 40 \mu \mathrm{W} \mathrm{cm}{ }^{-2}\right.$ ), respectively. $O D_{600}$ and $D s$ Red fluorescence were determined as described above. Data represent the mean \pm s.d. of four biologically independent replicates.

For the quantitative analysis of NmPAL binding to RNA, we recorded its interaction with 4 nM TAMRAlabeled 04.17 aptamer by fluorescence anisotropy as described before ${ }^{26}$. Experiments were carried out in reaction buffer containing 12 mM 4-(2-hydroxyethyl)-1-piperazineethanesulfonic acid (HEPES)/HCl pH 7.7, $135 \mathrm{mM} \mathrm{KCl}, 10 \mathrm{mM} \mathrm{NaCl}, 1 \mathrm{mM} \mathrm{MgCl}$, 10\% (v/v) glycerol, $100 \mu \mathrm{g} \mathrm{mL}^{-1} \mathrm{BSA}$. Fluorescence anisotropy was recorded with a multi-mode microplate reader (CLARIOstar) at (540 \pm 10$) \mathrm{nm}$ excitation, $(590 \pm 10) \mathrm{nm}$ emission, and using a 566-nm long-pass beam splitter. Data obtained in the presence of rising concentrations of either dark-adapted or light-adapted NmPAL (obtained by illumination with $455 \mathrm{~nm}, 50 \mathrm{~mW} \mathrm{~cm}^{-2}, 60 \mathrm{~s}$ ) were fitted to single-site binding isotherms using Fit-o-mat ${ }^{72}$ according to eq. (1).

$$
r=r_{0}+r_{1} \times[P A L] /\left([P A L]+K_{d}\right)
$$

To probe the light-dependent activity of NmPAL variants in eukaryotic cells, 50,000 Hela cells per well were seeded in 24 -well plate format ${ }^{26}$. Following $24 \mathrm{~h}$ incubation at $37^{\circ} \mathrm{C}$, cells were transfected. In brief, the medium was aspirated, and $500 \mu \mathrm{L}$ OptiMem medium were added to each well. In parallel, the transfection mix was prepared by combining $450 \mathrm{ng}$ plasmid encoding an mCherry-tagged NmPAL variant and $50 \mathrm{ng}$ reporter plasmid encoding Metridia secreted luciferase in $50 \mu \mathrm{L}$ OptiMem plus $2 \mu \mathrm{L}$ lipofectamin 2000. Upon incubation for $20 \mathrm{~min}$ at room temperature, $50 \mu \mathrm{L}$ of the transfection mix were added to each well, followed by incubation for $4 \mathrm{~h}$ at $37^{\circ} \mathrm{C}$ in either darkness or under blue light $\left(100 \mu \mathrm{W} \mathrm{cm}{ }^{-2}, 465 \mathrm{~nm}, 60 \mathrm{~s}\right.$ dark intervals followed by $30 \mathrm{~s}$ light intervals). The cell supernatant was then replaced by full medium (DMEM, supplemented with $10 \%$ fetal calf serum), and incubation continued at $37^{\circ} \mathrm{C}$. At $19 \mathrm{~h}$ post transfection, the luciferase expression was assessed by transferring $50 \mu \mathrm{L}$ of the cell supernatant to a fresh 96-well white plate (Lumitrac 200, Greiner). $5 \mu \mathrm{L}$ of the luciferase reagent (Ready-To-Glow secreted luciferase, Takara Clontech) were 
added to each well, and the plate was incubated for $25 \mathrm{~min}$ at room temperature. Chemiluminescence was then measured using an EnSpire plate reader (Perkin Elmer) with an integration time of $5 \mathrm{~s}$.

\section{Diguanylate cyclase assay}

The activity of the LOV-GGDEF protein was assessed in the E. coli strain KN78 which carries a knockout of the major diguanylate cyclase DgcE and encodes in its genome a translational fusion between the nucleator protein $\operatorname{csg} B$ involved in curli formation and the $\beta$-galactosidase $l a c Z^{62,75}$. To this end, a pQE-30 vector encoding a given LOV-GGDEF variant was transformed into E. coli. An empty PQE-30 plasmid served as negative control; as positive control, the empty PQE-30 plasmid was transformed into strain AR1100 which expresses a functional copy of DgcE. Bacterial starter cultures were grown overnight at $37^{\circ} \mathrm{C}$ in $5 \mathrm{~mL} \mathrm{LB}$ medium supplemented with $50 \mu \mathrm{g} \mathrm{mL}^{-1}$ ampicillin. Cultures were then diluted 100-fold, $1 \mathrm{mM}$ IPTG was added, and growth continued for $24 \mathrm{~h}$ at $28^{\circ} \mathrm{C}$ and $550 \mathrm{rpm}$ in either darkness, under constant blue light ( $450 \mathrm{~nm}, 40 \mu \mathrm{W} \mathrm{cm}{ }^{-2}$ ), or under constant red light $\left(660 \mathrm{~nm}, 40 \mu \mathrm{W} \mathrm{cm}{ }^{-2}\right.$ ). LacZ activity was then determined according to Miller ${ }^{76}$ using the chromogenic substrate ortho-nitrophenyl- $\beta$-galactoside. Data represent mean \pm s.d. of three separate experiments comprising four biologically independent replicates each.

\section{Structure determination of $A s L O V 2$ variants}

The expression vectors for the AsLOV2 variants were intentionally designed such that upon Senp2 cleavage during purification (see above) the same N-terminal GEF cloning artifact resulted as in a previous structural study ${ }^{28}$. Crystallization was conducted by sitting-drop vapor diffusion at solvent conditions adapted from the previous report ${ }^{28}$. Orthorhombic crystals were obtained at protein concentrations between 10 and $20 \mathrm{mg}$ $\mathrm{mL}^{-1}$ in $0.1 \mathrm{M}$ sodium acetate $\mathrm{pH}$ 4.6-5.0, 6-8\% (w/v) PEG 4000, 30\% (v/v) glycerol. Crystal growth and handling were generally performed in darkness or under dim red light, respectively. To characterize the darkadapted state, single crystals were mounted in loops and rapidly cryo-cooled by immersion in liquid nitrogen. To assess the light-adapted state, crystals were exposed to blue light ( $470 \mathrm{~nm}, 20 \mathrm{~mW} \mathrm{~cm}{ }^{-2}, 1 \mathrm{~min}$ ) prior to cryo-cooling. Diffraction data were collected at the BESSY (beamlines 14.1 and 14.2 ) synchrotron ${ }^{77}$ to resolutions between $0.90 \AA$ and $1.09 \AA$ (Suppl. Tables S1 and S2). Indexing and integration were performed with XDS $^{78}$, and scaling was done with Pointless ${ }^{79}$, all through the XDSapp interface ${ }^{80}$. Structures were solved by molecular replacement using the previously determined structure of dark-adapted AsLOV2 as search model (PDB entry $2 \mathrm{vOu}^{28}$ ). Model building was done in $\operatorname{Coot}^{81}$, and restrained refinement with anisotropic $B$ factors was conducted in Refmac ${ }^{82}$. Occupancies of residues with multiple conformations were manually refined. Due to the absence of electron density for the covalent thioadduct in the light-adapted structures, the cofactors were generally modelled as noncovalently bound oxidized flavin mononucleotides. Atom coordinates and structure-factor amplitudes were deposited in the Protein Data Bank under accession codes 7pgx (wildtype, dark), 7pgy (wild-type, light), 7pgz (Q513L, dark), and 7ph0 (Q513L, light). Molecular graphics were 
prepared with PyMOL (Schrodinger LLC). Root mean square deviation between the structures was calculated with LSQKAB ${ }^{83}$.

\section{Molecular simulations}

785

786

787

788

789

790

791

792

793

794

795

796

797

798

799

800

801

802

803

804

805

806

807

808

809

810

811

812

813

814

815

816

The simulations were performed using the crystal structures obtained in this work. Missing hydrogen atoms were added to the initial structures using the tleap program of AMBER 18. The protonation states of all titratable residues were considered at a pH of 7.0. The protein was solvated in a truncated octahedral box of TIP3P water molecules with a distance of at least $15 \AA$ between the atoms and the boundaries of the box. The system was neutralized by adding $\mathrm{K}^{+}$and $\mathrm{Cl}^{-}$ions. The SHAKE algorithm was used to constrain the bonds involving hydrogen atoms in all classical MD simulations, allowing a time step to be $2 \mathrm{fs}$. A Langevin thermostat with a collision frequency of $1 \mathrm{ps}^{-1}$ was used for temperature control in all simulations. The VMD plugin VolMap served to analyze the water density inside the protein. The MM parameters for FMN and the FMNCys adduct were obtained from ${ }^{84}$.

Initially, the solvent was minimized in 100,000 steps with restraints of $100 \mathrm{kcal} \mathrm{mol}^{-1} \AA^{-2}$ on all protein atoms and FMN. The system was then gradually heated from $100 \mathrm{~K}$ to $300 \mathrm{~K}$ within $50 \mathrm{~ns}$ with restraints on protein and FMN in NVT ensemble. The density of the solvent was then gradually equilibrated for another 20 ns under NPT conditions. The equilibration was extended for another 20 ns with weaker restraints of $10 \mathrm{kcal}$ $\mathrm{mol}^{-1} \AA^{-2}$. Then, MD of $20 \mathrm{~ns}$ each was conducted with weakened restraints of $1 \mathrm{kcal} \mathrm{mol}^{-1} \AA^{-2}$ and $0.1 \mathrm{kcal}$ $\mathrm{mol}^{-1} \AA^{-2}$, respectively, on the protein backbone. Finally, an unrestrained MD production run of 300 ns was carried out.

\section{Sequence analysis of LOV receptors lacking the active-site glutamine}

As in a previous analysis ${ }^{14}$, a BLAST search was performed with Bacillus subtilis YtvA (BsYtvA ${ }^{25}$, residues 1$127)$ as the query sequence and with an E-value cutoff of 10 . Using custom Python scripts, the results were filtered for entries that possess at least eight out of nine residues (residue positions Gly59, Asn61, Cys62, Arg63, Phe64, Leu65, Gln66, Asn94 and Asn104 in BsYtvA), which are conserved across LOV receptors ${ }^{29}$, but lack the active-site glutamine (position Gln123 in BsYtvA). Corresponding entries were aligned to the sequences of BsYtvA and AsLOV2 using ClustalX ${ }^{85}$. A sequence logo was generated with WebLogo version 3.7 86.

\section{Acknowledgements}

We thank U. Jenal for discussion; R. Hengge for discussion and supplying the $E$. coli reporter strains harboring the genomic $\operatorname{csgB}$ ::lacZ integration; and C. Feiler and F. Lennartz for assistance with data acquisition at the BESSY synchrotron. Funding by the Deutsche Forschungsgemeinschaft (grants MO2192/6-1/2 and M02192/8-1 to A.M.; grants MA3442/5-1/2 to G.M.) and the Alexander-von-Humboldt Foundation (SofjaKovalevskaya Award to A.M.) is gratefully acknowledged. 


\section{Authors' contributions}

J.D. performed all experiments on the YF1 and LOV ${ }^{\triangle Q}$-GGDEF variants. R.G. performed all experiments on the isolated AsLOV2 domain and refined crystal structures. J.K. analyzed NmPAL in bacterial reporter assays and by absorbance spectroscopy, and she studied its RNA interaction by fluorescence anisotropy. V.B. and I.S. conducted and evaluated molecular simulations. C.R., S.P., and G.M. did experiments on NmPAL in eukaryotic cells. A.T.R. performed spectroscopy on NmPAL and analyzed RNA binding. A.G.F. analyzed AsLOV2 variants by CD spectroscopy. T.G. and R.P.D. developed the fluorescence anisotropy assay for YF1. M.W. advised on crystallization and structure refinement. A.M. conducted sequence analyses, refined crystal structures, and conceived and coordinated the research. J.D. and A.M. wrote the manuscript with input from all authors.

827

\section{Conflict of interest}

829 The authors declare no conflict of interest.

830

831

\section{Data availability}

832 Atom coordinates and structure-factor amplitudes have been deposited in the Protein Data Bank under ac-

833 cession codes 7pgx (AsLOV2 wild-type, dark), 7pgy (wild-type, light), 7pgz (Q513L, dark), and 7ph0 (Q513L,

834 light). Other data are available from the authors upon request.

835

836

\section{References}

837

838

839

840

841

842

843

844

845

846

847

848

849

850

851

852

1. Christie, J. M. et al. Arabidopsis NPH1: a flavoprotein with the properties of a photoreceptor for phototropism. Science 282, 1698-1701 (1998).

2. Conrad, K. S., Manahan, C. C. \& Crane, B. R. Photochemistry of flavoprotein light sensors. Nat. Chem. Biol. 10, 801809 (2014).

3. Möglich, A., Yang, X., Ayers, R. A. \& Moffat, K. Structure and function of plant photoreceptors. Annu. Rev. Plant Biol. 61, 21-47 (2010).

4. Deisseroth, K. Optogenetics. Nat Methods 8, 26-29 (2011).

5. Losi, A., Gardner, K. H. \& Möglich, A. Blue-Light Receptors for Optogenetics. Chem. Rev. 118, 10659-10709 (2018).

6. Möglich, A., Ayers, R. A. \& Moffat, K. Structure and signaling mechanism of Per-ARNT-Sim domains. Structure 17, 1282-1294 (2009).

7. Crosson, S. \& Moffat, K. Structure of a flavin-binding plant photoreceptor domain: insights into light-mediated signal transduction. Proc Natl Acad Sci U A 98, 2995-3000 (2001).

8. Fedorov, R. et al. Crystal structures and molecular mechanism of a light-induced signaling switch: The Phot-LOV1 domain from Chlamydomonas reinhardtii. Biophys J 84, 2474-82 (2003).

9. Kottke, T., Heberle, J., Hehn, D., Dick, B. \& Hegemann, P. Phot-LOV1: photocycle of a blue-light receptor domain from the green alga Chlamydomonas reinhardtii. Biophys J 84, 1192-1201 (2003). 
10. Salomon, M., Christie, J. M., Knieb, E., Lempert, U. \& Briggs, W. R. Photochemical and mutational analysis of the FMN-binding domains of the plant blue light receptor, phototropin. Biochemistry 39, 9401-10 (2000).

11. Pfeifer, A. et al. Time-resolved Fourier transform infrared study on photoadduct formation and secondary structural changes within the phototropin LOV domain. Biophys. J. 96, 1462-1470 (2009).

12. Schleicher, E. et al. On the reaction mechanism of adduct formation in LOV domains of the plant blue-light receptor phototropin. J Am Chem Soc 126, 11067-76 (2004).

13. Alexandre, M. T. A., Arents, J. C., van Grondelle, R., Hellingwerf, K. J. \& Kennis, J. T. M. A Base-Catalyzed Mechanism for Dark State Recovery in the Avena sativa Phototropin-1 LOV2 Domain. Biochemistry 46, 3129-3137 (2007).

14. Yee, E. F. et al. Signal transduction in light-oxygen-voltage receptors lacking the adduct-forming cysteine residue. Nat. Commun. 6, 10079 (2015).

15. Harper, S. M., Neil, L. C. \& Gardner, K. H. Structural basis of a phototropin light switch. Science 301, 1541-1544 (2003).

16. Zoltowski, B. D. \& Crane, B. R. Light activation of the LOV protein vivid generates a rapidly exchanging dimer. Biochemistry 47, 7012-7019 (2008).

17. Berntsson, O. et al. Sequential conformational transitions and $\alpha$-helical supercoiling regulate a sensor histidine kinase. Nat. Commun. 8, 284 (2017).

18. Salomon, M. et al. An optomechanical transducer in the blue light receptor phototropin from Avena sativa. Proc. Natl. Acad. Sci. 98, 12357-12361 (2001).

19. Crosson, S. \& Moffat, K. Photoexcited structure of a plant photoreceptor domain reveals a light-driven molecular switch. Plant Cell 14, 1067-1075 (2002).

20. Nozaki, D. et al. Role of Gln1029 in the Photoactivation Processes of the LOV2 Domain in Adiantum Phytochrome3. Biochemistry 43, 8373-8379 (2004).

21. Nash, A. I., Ko, W.-H., Harper, S. M. \& Gardner, K. H. A conserved glutamine plays a central role in LOV domain signal transmission and its duration. Biochemistry 47, 13842-13849 (2008).

22. Ganguly, A., Thiel, W. \& Crane, B. R. Glutamine Amide Flip Elicits Long Distance Allosteric Responses in the LOV Protein Vivid. J. Am. Chem. Soc. 139, 2972-2980 (2017).

23. Freddolino, P. L., Dittrich, M. \& Schulten, K. Dynamic Switching Mechanisms in LOV1 and LOV2 Domains of Plant Phototropins. Biophys. J. 91, 3630-3639 (2006).

24. Iuliano, J. N. et al. Unraveling the Mechanism of a LOV Domain Optogenetic Sensor: A Glutamine Lever Induces Unfolding of the Ja Helix. ACS Chem. Biol. 15, 2752-2765 (2020).

25. Losi, A., Quest, B. \& Gärtner, W. Listening to the blue: the time-resolved thermodynamics of the bacterial blue-light receptor YtvA and its isolated LOV domain. Photochem Photobiol Sci 2, 759-66 (2003).

26. Weber, A. M. et al. A blue light receptor that mediates RNA binding and translational regulation. Nat Chem Biol 15, 1085-1092 (2019).

27. Zoltowski, B. D. et al. Conformational Switching in the Fungal Light Sensor Vivid. Science 316, 1054-1057 (2007).

28. Halavaty, A. S. \& Moffat, K. N- and C-terminal flanking regions modulate light-induced signal transduction in the LOV2 domain of the blue light sensor phototropin 1 from Avena sativa. Biochemistry 46, 14001-14009 (2007).

29. Crosson, S., Rajagopal, S. \& Moffat, K. The LOV domain family: photoresponsive signaling modules coupled to diverse output domains. Biochemistry 42, 2-10 (2003).

30. Pudasaini, A. et al. Steric and Electronic Interactions at Gln154 in ZEITLUPE Induce Reorganization of the LOV Domain Dimer Interface. Biochemistry 60, 95-103 (2021). 
894

31. Kobayashi, I., Nakajima, H. \& Hisatomi, O. Molecular Mechanism of Light-Induced Conformational Switching of the LOV Domain in Aureochrome-1. Biochemistry 59, 2592-2601 (2020).

32. He, L. et al. Circularly permuted LOV2 as a modular photoswitch for optogenetic engineering. Nat. Chem. Biol. 17, 915-923 (2021).

33. Polverini, E., Schackert, F. K. \& Losi, A. Interplay among the "flipping" glutamine, a conserved phenylalanine, water and hydrogen bonds within a blue-light sensing LOV domain. Photochem. Photobiol. Sci. 19, 892-904 (2020).

34. Zayner, J. P., Antoniou, C., French, A. R., Hause, R. J., Jr \& Sosnick, T. R. Investigating models of protein function and allostery with a widespread mutational analysis of a light-activated protein. Biophys. J. 105, 1027-1036 (2013).

35. Ohlendorf, R., Vidavski, R. R., Eldar, A., Moffat, K. \& Möglich, A. From dusk till dawn: one-plasmid systems for lightregulated gene expression. J. Mol. Biol. 416, 534-542 (2012).

36. Diensthuber, R. P., Bommer, M., Gleichmann, T. \& Möglich, A. Full-length structure of a sensor histidine kinase pinpoints coaxial coiled coils as signal transducers and modulators. Structure 21, 1127-1136 (2013).

37. Möglich, A., Ayers, R. A. \& Moffat, K. Design and signaling mechanism of light-regulated histidine kinases. J. Mol. Biol. 385, 1433-1444 (2009).

38. Zayner, J. P. \& Sosnick, T. R. Factors That Control the Chemistry of the LOV Domain Photocycle. PLoS ONE 9, e87074 (2014).

39. Iseki, M. et al. A blue-light-activated adenylyl cyclase mediates photoavoidance in Euglena gracilis. Nature 415, 1047-1051 (2002).

40. Gomelsky, M. \& Klug, G. BLUF: a novel FAD-binding domain involved in sensory transduction in microorganisms. Trends Biochem Sci 27, 497-500 (2002).

41. Pudasaini, A., El-Arab, K. K. \& Zoltowski, B. D. LOV-based optogenetic devices: light-driven modules to impart photoregulated control of cellular signaling. Front. Mol. Biosci. 2, 18 (2015).

42. Ziegler, T. \& Möglich, A. Photoreceptor engineering. Front. Mol. Biosci. 2, 30 (2015).

43. Hennemann, J. et al. Optogenetic Control by Pulsed Illumination. Chembiochem 19, 1296-1304 (2018).

44. Kawano, F., Aono, Y., Suzuki, H. \& Sato, M. Fluorescence Imaging-Based High-Throughput Screening of Fast- and Slow-Cycling LOV Proteins. PLoS ONE 8, e82693 (2013).

45. Nellen-Anthamatten, D. et al. Bradyrhizobium japonicum FixK2, a crucial distributor in the FixLJ-dependent regulatory cascade for control of genes inducible by low oxygen levels. J Bacteriol 180, 5251-5255 (1998).

46. Russo, F. D. \& Silhavy, T. J. The essential tension: opposed reactions in bacterial two-component regulatory systems. Trends Microbiol 1, 306-310 (1993).

47. Möglich, A. Signal transduction in photoreceptor histidine kinases. Protein Sci. 28, 1923-1946 (2019).

48. Harper, S. M., Christie, J. M. \& Gardner, K. H. Disruption of the LOV-Jalpha helix interaction activates phototropin kinase activity. Biochemistry 43, 16184-16192 (2004).

49. Harper, S. M., Neil, L. C., Day, I. J., Hore, P. J. \& Gardner, K. H. Conformational changes in a photosensory LOV domain monitored by time-resolved NMR spectroscopy. J Am Chem Soc 126, 3390-1 (2004).

50. Zayner, J. P., Antoniou, C. \& Sosnick, T. R. The amino-terminal helix modulates light-activated conformational changes in AsLOV2. J. Mol. Biol. 419, 61-74 (2012).

51. Wu, Y. I. et al. A genetically encoded photoactivatable Rac controls the motility of living cells. Nature 461, 104-108 (2009).

52. Strickland, D., Moffat, K. \& Sosnick, T. R. Light-activated DNA binding in a designed allosteric protein. Proc. Natl. Acad. Sci. U. S. A. 105, 10709-10714 (2008). 
53. Shu, X. et al. A Genetically Encoded Tag for Correlated Light and Electron Microscopy of Intact Cells, Tissues, and Organisms. PLOS Biol. 9, e1001041 (2011).

54. Kopka, B. et al. Electron transfer pathways in a light, oxygen, voltage (LOV) protein devoid of the photoactive cysteine. Sci. Rep. 7, 13346 (2017).

55. Möglich, A. \& Moffat, K. Structural Basis for Light-dependent Signaling in the Dimeric LOV Domain of the Photosensor YtvA. J Mol Biol 373, 112-126 (2007).

56. Yao, X., Rosen, M. K. \& Gardner, K. H. Estimation of the available free energy in a LOV2-J alpha photoswitch. Nat. Chem. Biol. 4, 491-497 (2008).

57. Vaidya, A. T., Chen, C.-H., Dunlap, J. C., Loros, J. J. \& Crane, B. R. Structure of a light-activated LOV protein dimer that regulates transcription. Sci. Signal. 4, ra50 (2011).

58. Dietler, J. et al. A Light-Oxygen-Voltage Receptor Integrates Light and Temperature. J. Mol. Biol. 433, 167107 (2021).

59. Conrad, K. S., Bilwes, A. M. \& Crane, B. R. Light-induced subunit dissociation by a light-oxygen-voltage domain photoreceptor from Rhodobacter sphaeroides. Biochemistry 52, 378-391 (2013).

60. Rivera-Cancel, G., Ko, W., Tomchick, D. R., Correa, F. \& Gardner, K. H. Full-length structure of a monomeric histidine kinase reveals basis for sensory regulation. Proc. Natl. Acad. Sci. U. S. A. 111, 17839-17844 (2014).

61. Jenal, U., Reinders, A. \& Lori, C. Cyclic di-GMP: second messenger extraordinaire. Nat. Rev. Microbiol. 15, 271-284 (2017).

62. Serra, D. O., Richter, A. M., Klauck, G., Mika, F. \& Hengge, R. Microanatomy at Cellular Resolution and Spatial Order of Physiological Differentiation in a Bacterial Biofilm. mBio 4, (2013).

63. Kalvaitis, M. E., Johnson, L. A., Mart, R. J., Rizkallah, P. \& Allemann, R. K. A Noncanonical Chromophore Reveals Structural Rearrangements of the Light-Oxygen-Voltage Domain upon Photoactivation. Biochemistry 58, 26082616 (2019).

64. Alexandre, M. T., van Grondelle, R., Hellingwerf, K. J. \& Kennis, J. T. Conformational heterogeneity and propagation of structural changes in the LOV2/Jalpha domain from Avena sativa phototropin 1 as recorded by temperaturedependent FTIR spectroscopy. Biophys J 97, 238-47 (2009).

65. Nash, A. I. et al. Structural basis of photosensitivity in a bacterial light-oxygen-voltage/helix-turn-helix (LOV-HTH) DNA-binding protein. Proc. Natl. Acad. Sci. U. S. A. 108, 9449-9454 (2011).

66. Remeeva, A. et al. Insights into the mechanisms of LOV domain color tuning from a set of high-resolution X-ray structures. bioRxiv 2021.02.05.429969 (2021) doi:10.1101/2021.02.05.429969.

67. Drepper, T. et al. Reporter proteins for in vivo fluorescence without oxygen. Nat. Biotechnol. 25, $443-445$ (2007).

68. Chapman, S. et al. The photoreversible fluorescent protein iLOV outperforms GFP as a reporter of plant virus infection. Proc. Natl. Acad. Sci. U. S. A. 105, 20038-20043 (2008).

69. Gibson, D. G. et al. Enzymatic assembly of DNA molecules up to several hundred kilobases. Nat. Methods 6, 343345 (2009).

70. Mathes, T., Vogl, C., Stolz, J. \& Hegemann, P. In vivo generation of flavoproteins with modified cofactors. J. Mol. Biol. 385, 1511-1518 (2009).

71. Andersen, K. R., Leksa, N. C. \& Schwartz, T. U. Optimized E. coli expression strain LOBSTR eliminates common contaminants from His-tag purification. Proteins Struct. Funct. Bioinforma. 81, 1857-1861 (2013).

72. Möglich, A. An Open-Source, Cross-Platform Resource for Nonlinear Least-Squares Curve Fitting. J. Chem. Educ. 95, 2273-2278 (2018). 
975
73. Ohlendorf, R., Schumacher, C. H., Richter, F. \& Möglich, A. Library-Aided Probing of Linker Determinants in Hybrid Photoreceptors. ACS Synth. Biol. 5, 1117-1126 (2016).

74. Strack, R. L. et al. A noncytotoxic DsRed variant for whole-cell labeling. Nat. Methods 5, 955-957 (2008).

75. Sommerfeldt, N. et al. Gene expression patterns and differential input into curli fimbriae regulation of all GGDEF/EAL domain proteins in Escherichia coli. Microbiology, 155, 1318-1331 (2009).

76. Miller, J. H. Experiments in Molecular Genetics. (Cold Spring Harbor Laboratory, Cold Spring Harbor, NY, 1972).

77. Mueller, U. et al. The macromolecular crystallography beamlines at BESSY II of the Helmholtz-Zentrum Berlin: Current status and perspectives. Eur. Phys. J. Plus 130, 141 (2015).

78. Kabsch, W. XDS. Acta Crystallogr. D Biol. Crystallogr. 66, 125-132 (2010).

79. Evans, P. R. An introduction to data reduction: space-group determination, scaling and intensity statistics. Acta Crystallogr. D Biol. Crystallogr. 67, 282-292 (2011).

80. Krug, M., Weiss, M. S., Heinemann, U. \& Mueller, U. XDSAPP: a graphical user interface for the convenient processing of diffraction data using XDS. J. Appl. Crystallogr. 45, 568-572 (2012).

81. Emsley, P. \& Cowtan, K. Coot: model-building tools for molecular graphics. Acta Crystallogr. D Biol. Crystallogr. 60, 2126-2132 (2004).

82. Murshudov, G. N., Vagin, A. A. \& Dodson, E. J. Refinement of macromolecular structures by the maximum-likelihood method. Acta Crystallogr Biol Crystallogr 53, 240-255 (1997).

83. Kabsch, W. A solution for the best rotation to relate two sets of vectors. Acta Cryst A 32, 922-923 (1976).

84. Bocola, M., Schwaneberg, U., Jaeger, K.-E. \& Krauss, U. Light-induced structural changes in a short light, oxygen, voltage (LOV) protein revealed by molecular dynamics simulations-implications for the understanding of LOV photoactivation. Front. Mol. Biosci. 2, 55 (2015).

85. Larkin, M. A. et al. Clustal W and Clustal X version 2.0. Bioinformatics 23, 2947-2948 (2007).

86. Crooks, G. E., Hon, G., Chandonia, J.-M. \& Brenner, S. E. WebLogo: A Sequence Logo Generator. Genome Res. 14, 1188-1190 (2004). 


\section{Supplementary Files}

This is a list of supplementary files associated with this preprint. Click to download.

- msQSupplMat.pdf

- 7pgxAsLOV2darkD1292117460valreportfullP1v12.pdf

- 7pgyAsLOV2lightD1292117461valreportfullP1v16.pdf

- 7pgzQ513LdarkD1292117462valreportfullP1v16.pdf

- 7ph0Q513LlightD1292117463valreportfullP1v13re.pdf 